\title{
Operational nonclassicality of local multipartite correlations in the limited-dimensional simulation scenario
}

\author{
C. Jebaratnam \\ E-mail: jebarathinam@bose.res.in \\ S. N. Bose National Centre for Basic Sciences, Salt Lake, Kolkata 700 098, India
}

\section{Debarshi Das}

E-mail: debarshidas@jcbose.ac.in

Centre for Astroparticle Physics and Space Science (CAPSS), Bose Institute, Block EN, Sector V, Salt Lake, Kolkata 700 091, India

\section{Suchetana Goswami}

S. N. Bose National Centre for Basic Sciences, Salt Lake, Kolkata 700 098, India

\author{
R. Srikanth \\ E-mail: srik@poornaprajna.org \\ Poornaprajna Institute of Scientific Research Bangalore- 560 080, Karnataka, \\ India
}

\author{
A. S. Majumdar \\ E-mail: archan@bose.res.in \\ S. N. Bose National Centre for Basic Sciences, Salt Lake, Kolkata 700 098, India \\ February 2014
}

\begin{abstract}
For a bipartite local quantum correlation, superlocality refers to the requirement for a larger dimension of the random variable in the classical simulation protocol than that of the quantum states that generate the correlations. In this work, we consider the classical simulation of local tripartite quantum correlations $P$ among three parties $A, B$ and $C$. If at least one of the bipartitions $(A \mid B C),(B \mid A C)$ and $(C \mid A B)$ is superlocal, then $P$ is said to be absolutely superlocal, whereas if all three bipartitions are superlocal, then $P$ is said to be genuinely superlocal. We present specific examples of genuine superlocality for tripartite correlations derived from three-qubit states. It is argued that genuine quantumness as captured by the notion of genuine discord is necessary for demonstrating genuine superlocality. Finally, the notions of absolute and genuine superlocality are also defined for multipartite correlations.
\end{abstract}




\section{Introduction}

Quantum composite systems exhibit nonclassical features such as entanglement [1] and Bell nonlocality [2]. In the Bell scenario, a correlation arising from local quantum measurements on a composite system is nonlocal if it cannot be simulated by sharing classical randomness, i.e., if it does not admit a local hidden variable (LHV) model [3]. It is well-known that quantum mechanics is not maximally nonlocal as there are nonsignaling (NS) correlations, e.g., Popescu-Rohrlich (PR) box [4], which are more nonlocal than that allowed by quantum theory. In the framework of generalized nonsignaling theory (GNST), there have been various attempts to find out the minimal constraints of quantum theory [5]. GNST has also been used in other contexts such as quantum cryptography [6] and quantifying nonlocality [7].

Recently, it has been shown that even some separable states demonstrate advantage in certain quantum information tasks if they have quantum discord which is a generalized measure of quantum correlations [8]. This initiated the study of nonclassicality going beyond nonlocality [9]. In Ref. [10], it has been shown that certain separable states which have quantumness may improve certain information theoretic protocols if there is a constraint on the possible local hidden variable models, i.e., shared randomness between the parties is limited to be finite. This provides a way for obtaining an operational meaning of the measures of quantumness such as quantum discord .

While entanglement is necessary for nonlocality, not all entangled states can be used to demonstrate nonlocality. In the context of classical simulation of local entangled states, Bowles et al [11] have defined a measure which is the minimal dimension of shared classical randomness that is needed to reproduce the statistics of a given local entangled state. Unlike the previous works, which used unbounded shared randomness to simulate a given local entangled state, Bowles et al have shown that all local entangled states can be simulated by using only finite shared randomness.

In Ref. [12], Donohue and Wolfe (DW) have provided upper bounds on the minimal dimension of the shared classical randomness required to simulate any local correlation in a given Bell scenario. DW have demonstrated an interesting feature of certain local boxes called superlocality: there exist local boxes which can be simulated by certain quantum systems of local dimension lower than that of the shared classical randomness needed to simulate them. That is, superlocality refers to the dimensional advantage in simulating certain local boxes by using quantum systems. In particular, DW [12] and also Goh et al [13] have shown that entanglement enables superlocality, however, superlocality occurs even for separable states.

In Ref. [14], Jebaratnam et al have pointed out that superlocality cannot occur for arbitrary separable states. In particular, Jebaratnam et al have argued that separable states which are a classical-quantum state [15] or its permutation can never lead to superlocality. Note that bipartite quantum states which are not a classical-quantum state must have quantumness as quantified by quantum discord [8]. The observation that the limited dimensional quantum simulation of certain local correlations requires quantumness in the states motivated the study of nonclassicality going beyond Bell nonlocality [16]. In Ref. [14], Jebaratnam et al have considered a measure of nonclassicality in the context of GNST called Bell strength for a family of local correlations and identified nonzero Bell strength as a quantification of superlocality as well.

The extension of the Bell-type scenario to more than two parties was first 
elaborated by Greenberger, Horne, and Zeilinger [17]. In Refs. [18-21], certain interesting features of nonlocality of tripartite systems over and above what is found for bipartite ones have been established. Recently, genuine multipartite quantum discord has been defined to quantify the quantumness shared among all subsystems of the multipartite quantum state [22-25]. In Ref. [26], Jebaratnam has demonstrated that the limited dimensional quantum simulation of certain local tripartite correlations must require genuine tripartite quantum discord states. To study genuine nonclassicality of these correlations, Jebaratnam has introduced two quantities called, Svetlichny strength and Mermin strength, in the context of tripartite nonsignaling boxes (i.e., correlations in the tripartite GNST).

For the case of multipartite systems, early studies have established that certain nonlocal measures may indeed be amplified by the addition of system dimensions [2730]. Multipartite quantum entanglement, however, displays complicated structures which can be broadly classified according to whether entanglement is shared among all the subsystems of a given multipartite system or not. In Ref. [18], Svetlichny introduced the notion of genuine multipartite nonlocality and derived Bell-type inequalities to detect it. Quite generally, genuine multipartite nonlocalty for a multipartite system occurs precisely when every bi-partition is nonlocal. By contrast, absolute nonlocality occurs when at least one bi-partition is nonlocal. Absolute multipartite nonlocality is indicated by the violation of a Mermin inequality [19].

In the present work, we are interested in providing an operational characterization of genuine and absolute nonclassicality via superlocality in the scenario of local tripartite systems and its generalization to higher-particle systems. It is argued that genuine quantum discord is necessary for demonstrating genuine superlocality. This implies that genuine superlocality provides an operational characterization of genuine quantum discord. The concept of superlocality is generalized for multipartite correlations also. As specific examples, we consider tripartite correlations that have non-vanishing Svetlichny strength or Mermin strength [26].

The organization of the paper is as follows. In Sec. 2, we present the mathematical tool that we use for the purpose of studying superlocality of local tripartite boxes, namely a polytope of tripartite nonsignaling boxes with two-binary-inputs-two-binaryoutputs. In Sec. 3, we define absolute and genuine superlocality for tripartite boxes and we present examples of two families of tripartite local correlations that are absolutely superlocal or genuinely superlocal, having their nonclassicality quantified in terms of nonzero Svetlichny strength and nonzero Mermin strength, respectively. In Sec. 4, we demonstrate the connection between genuine superlocality and genuine quantum discord. In Sec. 5, we generalize the notion of superlocality to $n$-partite correlations. Sec. 6 is reserved for certain concluding remarks.

\section{Preliminaries}

We are interested in quantum correlations arising from the following tripartite Bell scenario. Three spatially separated parties, say Alice, Bob, and Charlie, perform three dichotomic measurements on a shared tripartite quantum state $\rho \in \mathcal{H}_{A} \otimes \mathcal{H}_{B} \otimes \mathcal{H}_{C}$, where $\mathcal{H}_{K}$ denotes Hilbert space of $k$ th party. In this scenario, a correlation between the outcomes is described by the set of conditional probability distributions $P(a b c \mid x y z)$, where $x, y$, and $z$ denote the inputs (measurement choices) and $a, b$ and $c$ denote the outputs (measurement outcomes) of Alice, Bob and Charlie respectively (with $x, y, z, a, b, c \in\{0,1\}$ ). Suppose $M_{A_{x}}^{a}, M_{B_{y}}^{b}$ and $M_{C_{z}}^{c}$ denote the measurement 
operators of Alice, Bob, and Charlie, respectively. Then the correlation can be expressed through the Born's rule as follows:

$$
P(a b c \mid x y z)=\operatorname{Tr}\left(\rho M_{A_{x}}^{a} \otimes M_{B_{y}}^{b} \otimes M_{C_{z}}^{c}\right) .
$$

In particular, we focus on the scenario where the parties share a three-qubit state and perform spin projective measurements $A_{x}=\hat{a}_{x} \cdot \vec{\sigma}, B_{y}=\hat{b}_{y} \cdot \vec{\sigma}$, and $C_{z}=\hat{c}_{z} \cdot \vec{\sigma}$. Here $\hat{a}_{x}, \hat{b}_{y}$, and $\hat{c}_{z}$ are unit Bloch vectors denoting the measurement directions and $\vec{\sigma}=\left\{\sigma_{1}, \sigma_{2}, \sigma_{3}\right\}$, with $\left\{\sigma_{i}\right\}_{i=1,2,3}$ being the Pauli matrices.

The set of nonsignaling (NS) boxes with two binary inputs and two binary outputs forms a convex polytope $\mathcal{N}$ in a 26 dimensional space [31] and includes the set of quantum correlations $Q$ as a proper subset. Any box belonging to this polytope can be fully specified by 6 single-party, 12 two-party and 8 three-party expectations,

$$
\begin{aligned}
& P(a b c \mid x y z)=\frac{1}{8}\left[1+(-1)^{a}\left\langle A_{x}\right\rangle+(-1)^{b}\left\langle B_{y}\right\rangle+(-1)^{c}\left\langle C_{z}\right\rangle\right. \\
& +(-1)^{a \oplus b}\left\langle A_{x} B_{y}\right\rangle+(-1)^{a \oplus c}\left\langle A_{x} C_{z}\right\rangle+(-1)^{b \oplus c}\left\langle B_{y} C_{z}\right\rangle \\
& \left.+(-1)^{a \oplus b \oplus c}\left\langle A_{x} B_{y} C_{z}\right\rangle\right],
\end{aligned}
$$

where $\left\langle A_{x}\right\rangle=\sum_{a}(-1)^{a} P(a \mid x),\left\langle A_{x} B_{y}\right\rangle=\sum_{a, b}(-1)^{a \oplus b} P(a b \mid x y)$ and $\left\langle A_{x} B_{y} C_{z}\right\rangle=$ $\sum_{a, b, c}(-1)^{a \oplus b \oplus c} P(a b c \mid x y z), \oplus$ denotes modulo sum 2 . The set of boxes that can be simulated by a fully LHV model are of the form,

$$
P(a b c \mid x y z)=\sum_{\lambda=0}^{d_{\lambda}-1} p_{\lambda} P_{\lambda}(a \mid x) P_{\lambda}(b \mid y) P_{\lambda}(c \mid z),
$$

with $\sum_{\lambda=0}^{d_{\lambda}-1} p_{\lambda}=1$ and it forms the fully local (or, 3-local) polytope [32,33] denoted by $\mathcal{L}$. Here $\lambda$ denotes shared classical randomness which occurs with probability $p_{\lambda}$. For a given fully local box, the form (3) determines a classical simulation protocol with dimension $d_{\lambda}$ [12]. The extremal boxes of $\mathcal{L}$ are 64 local vertices which are fully deterministic boxes,

$$
P_{D}^{\alpha \beta \gamma \epsilon \zeta \eta}(a b c \mid x y z)=\left\{\begin{array}{rr}
1, & a=\alpha x \oplus \beta \\
& b=\gamma y \oplus \epsilon \\
& c=\zeta z \oplus \eta \\
0, & \text { otherwise. }
\end{array}\right.
$$

Here, $\alpha, \beta, \gamma, \epsilon, \zeta, \eta \in\{0,1\}$. The above boxes can be written as the product of deterministic distributions corresponding to Alice and Bob-Charlie, i.e., $P_{D}^{\alpha \beta \gamma \epsilon \zeta \eta}(a b c \mid x y z)=P_{D}^{\alpha \beta}(a \mid x) P_{D}^{\gamma \epsilon \zeta \eta}(b c \mid y z)$, here

$$
P_{D}^{\alpha \beta}(a \mid x)=\left\{\begin{array}{lr}
1, & a=\alpha x \oplus \beta \\
0, & \text { otherwise }
\end{array}\right.
$$

and

$$
P_{D}^{\gamma \epsilon \zeta \eta}(b c \mid y z)=\left\{\begin{array}{cc}
1, & b=\gamma y \oplus \epsilon \\
& c=\zeta z \oplus \eta \\
0, & \text { otherwise }
\end{array}\right.
$$


which can also be written as the product deterministic distributions corresponding to Bob and Charlie, i.e., $P_{D}^{\gamma \epsilon \zeta \eta}(b c \mid y z)=P_{D}^{\gamma \epsilon}(b \mid y) P_{D}^{\zeta \eta}(c \mid z)$, where

$$
P_{D}^{\gamma \epsilon}(b \mid y)=\left\{\begin{array}{lr}
1, & b=\gamma y \oplus \epsilon \\
0, & \text { otherwise }
\end{array}\right.
$$

and

$$
P_{D}^{\zeta \eta}(c \mid z)= \begin{cases}1, & c=\zeta z \oplus \eta \\ 0, & \text { otherwise. }\end{cases}
$$

Note that the set of 3-local boxes and quantum boxes satisfy $\mathcal{L} \subset Q \subset \mathcal{N}$. Boxes lying outside $\mathcal{L}$ are called absolutely nonlocal boxes and they cannot be written as a convex mixture of the local deterministic boxes alone.

A tripartite correlation is said to be "2-local across the bipartite cut $(A B \mid C)$ " if it has the following form:

$$
P(a b c \mid x y z)=\sum_{\lambda} p_{\lambda} P_{\lambda}(a b \mid x y) P_{\lambda}(c \mid z),
$$

where $P_{\lambda}(a b \mid x y)$ can have arbitrary nonlocality consistent with the NS principle. "2locality across other bipartite cuts" for tripartite correlations can be defined similarly. Note that any 3-local box can always be written in 2-local form across any possible bipartition. The general 2-local form [34] is, therefore:

$$
\begin{aligned}
P(a b c \mid x y z) & =s_{1} \sum_{\lambda} p_{\lambda} P_{\lambda}^{A B \mid C}+s_{2} \sum_{\lambda} q_{\lambda} P_{\lambda}^{A C \mid B} \\
& +s_{3} \sum_{\lambda} r_{\lambda} P_{\lambda}^{A \mid B C}
\end{aligned}
$$

where $P_{\lambda}^{A B \mid C}=P_{\lambda}(a b \mid x y) P_{\lambda}(c \mid z)$, and, where $P_{\lambda}^{A C \mid B}$ and $P_{\lambda}^{A \mid B C}$ are similarly defined. Here $s_{1}+s_{2}+s_{3}=1 ; \sum_{\lambda} p_{\lambda}=1 ; \sum_{\lambda} q_{\lambda}=1 ; \sum_{\lambda} r_{\lambda}=1$. Each bipartite distribution in the decomposition (10) can have arbitrary nonlocality consistent with the NS principle. A tripartite nonlocal box is genuinely tripartite nonlocal if it cannot be written in the 2-local form given by Eq. (10). Hence, a genuinely tripartite nonlocal box is nonlocal with respect to every bipartition $(A \mid B C),(B \mid C A),(C \mid A B)$.

The set of boxes that admit a decomposition as in Eq. (10) again forms a convex polytope which is called 2-local polytope, denoted by $\mathcal{L}_{2}$. The extremal boxes of this polytope are the 64 local vertices and 48 2-local vertices. There are 162 -local vertices in which a PR-box [4] is shared between $A$ and $B$,

$$
\begin{aligned}
& P_{12}^{\alpha \beta \gamma \epsilon}(a b c \mid x y z) \\
& =\left\{\begin{array}{lll}
\frac{1}{2}, & a \oplus b=x \cdot y \oplus \alpha x \oplus \beta y \oplus \gamma \quad \& \quad \begin{array}{l}
c=\gamma z \oplus \epsilon \\
0,
\end{array} & \text { otherwise, }
\end{array}\right.
\end{aligned}
$$

and the other 32 two-way local vertices, $P_{13}^{\alpha \beta \gamma \epsilon}$ and $P_{23}^{\alpha \beta \gamma \epsilon}$, in which a PR-box is shared by $A C$ and $B C$, are similarly defined. The extremal boxes in Eq. (11) can be written in the factorized form, $P_{12}^{\alpha \beta \gamma \epsilon}(a b c \mid x y z)=P_{P R}^{\alpha \beta \gamma}(a b \mid x y) P_{D}^{\gamma \epsilon}(c \mid z)$, where

$$
\begin{aligned}
& P_{P R}^{\alpha \beta \gamma}(a b \mid x y) \\
& =\left\{\begin{array}{lr}
\frac{1}{2}, & a \oplus b=x . y \oplus \alpha x \oplus \beta y \oplus \gamma \\
0, & \text { otherwise, }
\end{array}\right.
\end{aligned}
$$


and $P_{D}^{\gamma \epsilon}(c \mid z)$ is the aforementioned deterministic box. The set of 2-local boxes satisfy, $\mathcal{L} \subset \mathcal{L}_{2} \subset \mathcal{N}$. Absolute tripartite nonlocal boxes can be either 2-local or genuinely tripartite nonlocal. A genuinely tripartite nonlocal box cannot be written as a convex mixture of the extremal boxes of $\mathcal{L}_{2}$ and violates a facet inequality of $\mathcal{L}_{2}$ given in Ref. [34].

The Svetlichny inequalities [18] which are given by

$$
\mathcal{S}_{\alpha \beta \gamma \epsilon}=\sum_{x y z}(-1)^{x \cdot y \oplus x \cdot z \oplus y \cdot z \oplus \alpha x \oplus \beta y \oplus \gamma z \oplus \epsilon}\left\langle A_{x} B_{y} C_{z}\right\rangle \leq 4,
$$

are one of the classes of facet inequalities of the 2-local polytope. The violation of a Svetlichny inequality implies one of the forms of genuine tripartite nonlocality [34]. The following extremal tripartite nonlocal boxes:

$$
\begin{aligned}
& P_{\mathrm{Sv}}^{\alpha \beta \gamma \epsilon}(a b c \mid x y z) \\
& =\left\{\begin{array}{lr}
\frac{1}{4}, & a \oplus b \oplus c=x \cdot y \oplus x \cdot z \oplus y \cdot z \oplus \alpha x \oplus \beta y \oplus \gamma z \oplus \epsilon \\
0, & \text { otherwise, }
\end{array}\right.
\end{aligned}
$$

which violate a Svetlichny inequality to its algebraic maximum are called Svetlichny boxes.

Mermin inequalities [19] are one of the classes of facet inequalities of the fully local (or, 3-local) polytope [35,36]. One of the Mermin inequalities is given by,

$$
\left\langle A_{0} B_{0} C_{0}\right\rangle-\left\langle A_{0} B_{1} C_{1}\right\rangle-\left\langle A_{1} B_{0} C_{1}\right\rangle-\left\langle A_{1} B_{1} C_{0}\right\rangle \leq 2,
$$

and the other 15 Mermin inequalities can be obtained from the above inequality by local reversible operations which are analogous to local unitary operations in quantum theory and include local relabeling of the inputs and outputs. All the 16 Mermin inequalities are given by

$$
\mathcal{M}_{\alpha \beta \gamma \epsilon}=(\alpha \oplus \beta \oplus \gamma \oplus 1) \mathcal{M}_{\alpha \beta \gamma \epsilon}^{+}+(\alpha \oplus \beta \oplus \gamma) \mathcal{M}_{\alpha \beta \gamma \epsilon}^{-} \leq 2,
$$

where $\mathcal{M}_{\alpha \beta \gamma \epsilon}^{+}:=(-1)^{\gamma \oplus \epsilon}\left\langle A_{0} B_{0} C_{1}\right\rangle+(-1)^{\beta \oplus \epsilon}\left\langle A_{0} B_{1} C_{0}\right\rangle+(-1)^{\alpha \oplus \epsilon}\left\langle A_{1} B_{0} C_{0}\right\rangle+$ $(-1)^{\alpha \oplus \beta \oplus \gamma \oplus \epsilon \oplus 1}\left\langle A_{1} B_{1} C_{1}\right\rangle$ and

$\mathcal{M}_{\alpha \beta \gamma \epsilon}^{-}:=(-1)^{\alpha \oplus \beta \oplus \epsilon \oplus 1}\left\langle A_{1} B_{1} C_{0}\right\rangle+(-1)^{\alpha \oplus \gamma \oplus \epsilon \oplus 1}\left\langle A_{1} B_{0} C_{1}\right\rangle+(-1)^{\beta \oplus \gamma \oplus \epsilon \oplus 1}\left\langle A_{0} B_{1} C_{1}\right\rangle+$ $(-1)^{\epsilon}\left\langle A_{0} B_{0} C_{0}\right\rangle$. Mermin inequalities detect absolute nonlocality, i.e., it guarantees the box to lie outside $\mathcal{L}$. Quantum correlations that violate a Mermin inequality to its algebraic maximum demonstrate Greenberger-Horne-Zeilinger (GHZ) paradox [17] and are called Mermin boxes.

In the tripartite case, while the dimension of the NS polytope $\mathcal{N}$ is 26 , the number of extreme boxes are 53,856 [37]. Thus, a given $P$, expressed as a convex combination of the extreme boxes, does not have a unique decomposition. The correlations $P$ of interest to us, belong to a subpolytope of $\mathcal{N}$, called Svetlichny-box polytope in Ref. [26], denoted $\mathcal{R}$, having 128 extreme boxes (64 3-local boxes, 48 2-local boxes that are not 3-local, and finally, 16 Svetlichny boxes). The Svetlichny-box polytope can be seen as a generalization of the bipartite PR-box polytope.

Even in the case of the polytope $\mathcal{R}$, since it has a more extreme boxes than its dimension of 26 , therefore it has no unique decomposition. However, a unique, canonical, decomposition for any $P \in \mathcal{R}$ can be given, as shown in Ref. [26], by making use of the non-trivial symmetry properties among the extreme boxes of $\mathcal{R}$. 
Thereby, any nonlocal box $P \in \mathcal{R}$ violating a Svetlichny inequality can be brought to the form [26]:

$$
P=p_{S v} P_{S v}^{\alpha \beta \gamma \epsilon}+\left(1-p_{S v}\right) P_{S v L},
$$

where a single Svetlichny-box, $P_{S v}^{\alpha \beta \gamma \epsilon}$, is dominant and $p_{S v}$ has been maximized and $P_{S v L}$ is a Svetlichny-local box.

Eq. (17) is called the canonical decomposition for any box $P$ belonging to the Svetlichny-box polytope. Following Ref. [26], we refer to $p_{S v}$ in the canonical decomposition as Svetlichny strength. In a given situation, suppose $\mathbf{P}_{\mathrm{Sv}}^{\uparrow}$ denotes the dominant Svetlichny-box. The canonical form (17) becomes

$$
P=\mu \mathbf{P}_{\mathrm{Sv}}^{\uparrow}+(1-\mu) P_{S v L}^{\mathcal{G}=0} .
$$

The fact that $\mu$ represents the maximal fraction of $\mathbf{P}_{\mathrm{Sv}}^{\uparrow}$ over all decompositions has the following consequence. The quantity $\mathcal{G}$ in Eq. (18) is given by

$$
\mathcal{G}:=\min \left\{\mathcal{G}_{1}, \ldots, \mathcal{G}_{9}\right\}
$$

where $\mathcal{G}_{1}:=||\left|\mathcal{S}_{000}-\mathcal{S}_{001}\right|-\left|\mathcal{S}_{010}-\mathcal{S}_{011}\right||-|\left|\mathcal{S}_{100}-\mathcal{S}_{101}\right|-\left|\mathcal{S}_{110}-\mathcal{S}_{111}\right|||$ and the other eight $\mathcal{G}_{i}$ are similarly defined and can be obtained by interchanging $\mathcal{S}_{\alpha \beta \gamma}$ 's in $\mathcal{G}_{1}$. The motivation for this quantity is the "monoandrous" nature of Svetlichny boxes, whereby they are maximal for precisely one of the Svetlichny inequalities. On the other hand, the deterministic boxes have the same absolute values. As a result, $\mathcal{G}$ can be used to witness the canonicality of the decomposition, by checking that the local part has $\mathcal{G}=0[26]$.

The Svetlichny strength $\mu$ of the box given by Eq. (18) satisfies the relationship $\mathcal{G}(P)=8 \mu$ as shown in Ref. [26]. The box $P_{S v L}^{\mathcal{G}=0}$ in Eq.(18) is a Svetlichny-local box having $\mathcal{G}(P)=0$. Thus, the canonical decomposition (18) is irreducible in the sense that the full weight of genuine tripartite nonlocality has been transferred to the $\mathbf{P}_{\mathrm{Sv}}^{\uparrow}$ part and no further reduction of this weight is possible in the local part.

Analogously, it can be shown (see Section 3.2) that the canonical decomposition of $P_{S v L}$ in Eq. (18) wherein $p_{S v}=0$ is one which is a convex combination of a dominant Mermin box (an equal mixture of two Svetlichny boxes) and a Merminlocal box (one that doesn't violate the Mermin inequality), such that the weight of the dominant Mermin box has been maximized. Following Ref. [26], we define this maximized weight as Mermin strength of the correlation.

Accordingly, the Svetlichny-local part in Eq. (18), which is multiply decomposable, can itself be canonically decomposed such that the weight of the Mermin part has been maximized. Therefore, the boxes that we consider in this work belong to the family of boxes which have the following canonical form:

$$
P=\mu \mathbf{P}_{\mathrm{Sv}}^{\uparrow}+\nu \mathbf{P}_{\mathrm{M}}^{\uparrow}+(1-\mu-\nu) P_{S v L}^{\mathcal{G}=\mathcal{Q}=0}
$$

where $\mathbf{P}_{\mathrm{M}}^{\uparrow}$ is the dominant Mermin-box. The quantity $\mathcal{Q}$ in Eq. (20), which witnesses the canonicality of the Svetlichny-local decomposition into a Mermin-nonlocal and Mermin-local part, is given by

$$
Q:=\min \left\{Q_{1}, \ldots, Q_{9}\right\}
$$

where $Q_{1}=||\left|\mathcal{M}_{000}-\mathcal{M}_{001}\right|-\left|\mathcal{M}_{010}-\mathcal{M}_{011}\right||-|\left|\mathcal{M}_{100}-\mathcal{M}_{101}\right|-\left|\mathcal{M}_{110}-\mathcal{M}_{111}\right|||$, and other $Q_{i}$ 's are obtained by permutations (Here, $\mathcal{M}_{\alpha \beta \gamma}$ are given by Eq. (16)). The 
Mermin strength $\nu$ of the box given by Eq. (20) satisfies the relationship $\mathcal{Q}(P)=4 \nu$ as shown in Ref. [26]. The box $P_{S v L}^{\mathcal{G}=\mathcal{Q}=0}$ in Eq.(20) is a box having $\mathcal{G}(P)=0$ and $Q(P)=0$. Unlike the nonlocal cost [7], Svetlichny strength and/or Mermin strength can also be nonzero for certain fully local as well as 2-local correlations.

In the following, we study the non-classicality as captured by the notion of superlocality of two families of fully local and 2-local tripartite correlations which have Svetlichny strength and Mermin strength, respectively.

\section{Superlocality of tripartite fully local and 2-local boxes}

For a given local bipartite or $n$-partite box, let $d_{\lambda}$ denote the minimal dimension of the shared classical randomness. In Ref. [12], DW have derived the upper on $d_{\lambda}$ with the assumption that $n-1$ of the parties' measurements depend deterministically on $\lambda$, whereas the other party can use nondeterministic strategy on each $\lambda$ in the classical simulation model. Before we define superlocality for fully local tripartite boxes, let us define superlocality for local bipartite boxes.

Definition. Suppose we have a quantum state in $\mathbb{C}^{d^{A}} \otimes \mathbb{C}^{d^{B}}$ and measurements which produce a local bipartite box $P(a b \mid x y)$. The correlation $P$ is sublocal precisely if

$$
P(a b \mid x y)=\sum_{\lambda=0}^{d_{\lambda}-1} p_{\lambda} P_{\lambda}(a \mid x) P_{\lambda}(b \mid y),
$$

with $d_{\lambda} \leq d$, with $d=\min \left\{d_{A}, d_{B}\right\}$. Then, superlocality holds iff there is no sublocal decomposition of the given box. Here, $\sum_{\lambda=0}^{d_{\lambda}-1} p_{\lambda}=1$.

Note that, suppose the given local box is produced by a quantum state in $\mathbb{C}^{d_{A}} \otimes \mathbb{C}^{d_{B}}$. Then it can also be produced by a quantum state in $\mathbb{C}^{d} \otimes \mathbb{C}^{d}$ with $d=\min \left\{d_{A}, d_{B}\right\}$ [12].

Note that a bipartite correlation that is nonlocal is obviously non-sublocal because there is no dimensionally bounded preshared randomness that can reproduce it in a classical simulation protocol. The interesting cases where superlocality can witness quantumness will then pertain only to local correlations. Hence in this work, we shall be mainly concerned with superlocality as applied to local correlations.

We can now extend superlocality to a tripartite system.

Definition. Suppose we have a quantum state in $\mathbb{C}^{d^{A}} \otimes \mathbb{C}^{d^{B}} \otimes \mathbb{C}^{d^{C}}$ and measurements which produce a fully local tripartite box $P(a b c \mid x y z)$. The correlation $P$ is 3 -sublocal (or, fully sublocal) precisely if

$$
P(a b c \mid x y z)=\sum_{\lambda=0}^{d_{\lambda}-1} p_{\lambda} P_{\lambda}(a \mid x) P_{\lambda}(b \mid y) P_{\lambda}(c \mid z),
$$

with $d_{\lambda} \leq d$, with $d=\min \left\{d_{A}, d_{B}, d_{C}\right\}$. Then, absolute tripartite superlocality holds iff there is no 3 -sublocal decomposition of the given fully local box. Here, $\sum_{\lambda=0}^{d_{\lambda}-1} p_{\lambda}=1$.

In other words, if a fully local correlation $P(a b c \mid x y z)$ is such that at least one of the bipartitions $(A \mid B C),(B \mid A C)$ and $(C \mid A B)$ is superlocal, then $P(a b c \mid x y z)$ is said to have absolute tripartite superlocality. The set of all fully sublocal tripartite boxes forms a nonconvex subset of the fully local polytope $\mathcal{L}$ and is denoted by $\mathcal{L}_{3}^{*} \equiv \mathcal{L}^{*}$.

We now define the concept of 2-sublocality across a given bipartite cut. 


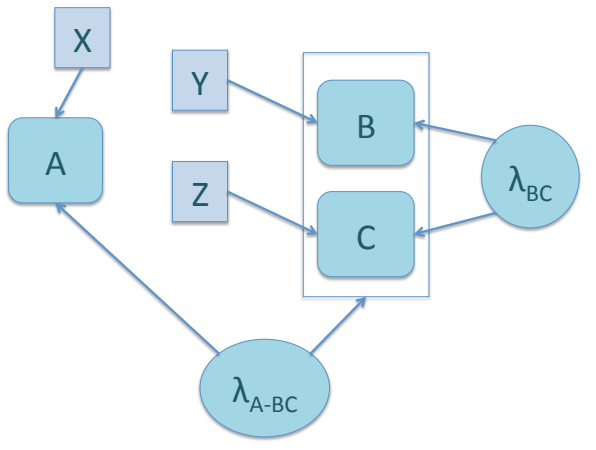

Figure 1. A 2-sublocal box: Directional acyclic graph illustrating simulation of a local tripartite box in the following classical protocol: Alice shares hidden variable $\lambda_{A-B C}$ with Bob-Charlie. For each $\lambda_{A-B C}$, Bob and Charlie can use shared randomness $\lambda_{B C}$ of arbitrary dimension.

Definition. Suppose we have a quantum state in $\mathbb{C}^{d^{A}} \otimes \mathbb{C}^{d^{B}} \otimes \mathbb{C}^{d^{C}}$ and measurements producing a tripartite box $P(a b c \mid x y z)$, which is 2-local across the bipartite cut $(A \mid B C)$. Then, the correlation $P$ is 2-sublocal across the bipartite cut $(A \mid B C)$ precisely if

$$
P(a b c \mid x y z)=\sum_{\lambda=0}^{d_{\lambda}-1} p_{\lambda} P_{\lambda}(a \mid x) P_{\lambda}(b c \mid y z),
$$

where $\sum_{\lambda=0}^{d_{\lambda}-1} p_{\lambda}=1 ; d_{\lambda} \leq \min \left\{d_{A}, d_{B} d_{C}\right\}$. Here, $P_{\lambda}(b c \mid y z)$ is an arbitrary NS box (If $P_{\lambda}(b c \mid y z)$ is local box, then $P(a b c \mid x y z)$ is fully local). Tripartite superlocality across the bipartite cut $(A \mid B C)$ holds iff there is no 2-sublocal decomposition across the bipartite cut $(A \mid B C)$.

Tripartite superlocality across other possible bipartite cuts can be defined in a similar way. The nonconvex set of all 2 -sublocal boxes across different possible bipartite cuts denoted $\mathcal{L}_{2}^{*}$ satisfies $\mathcal{L}^{*} \subset \mathcal{L}_{2}^{*}$.

Now we define genuine tripartite superlocality.

Definition. A tripartite local (fully local or 2-local) correlation box is called genuinely superlocal iff it is superlocal across all possible bipartitions.

A fully local correlation $P(a b c \mid x y z)$ that isn't 3 -sublocal has absolute superlocality. In such a correlation, there is at least one partition for which 2sublocality doesn't hold. Obviously, a fully local correlation that is genuinely superlocal must be absolutely superlocal also. Here we may remark that genuine superlocality can occur for absolutely nonlocal (i.e., which are not fully local), but 2-local correlations.

Two different classical simulation protocols of tripartite 2-local boxes (they may be fully local as well) are depicted in Fig. 1 and Fig. 2. Fig. 1 is applicable when the 2-local tripartite box is fully local as well; on the other hand, Fig. 2 is applicable when the 2-local tripartite box may or may not be fully local. We also use the notation $\lambda$ to denote $\lambda_{A-B C}$ for our convenience in calculations. 


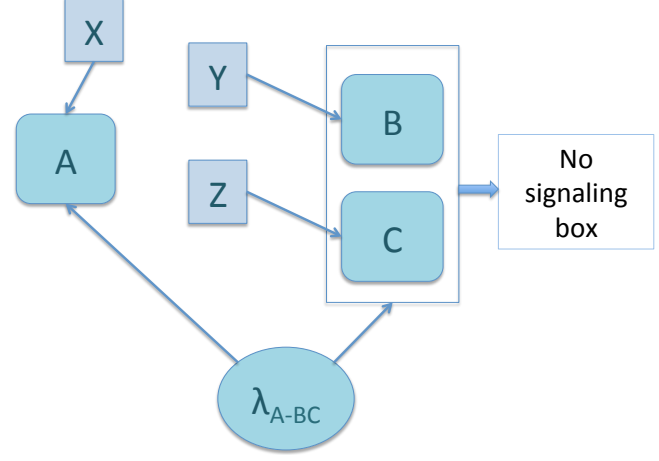

Figure 2. Directional acyclic graph illustrating simulation of a 2-local tripartite box in the following classical protocol: Alice shares hidden variable $\lambda_{A-B C}$ with Bob-Charlie. For each $\lambda_{A-B C}$, the correlations of Bob and Charlie are NS boxes.

\subsection{Noisy Svetlichny-box}

The generalized GHZ (GGHZ) state in $\mathbb{C}^{2} \otimes \mathbb{C}^{2} \otimes \mathbb{C}^{2}$,

$$
\left|\psi_{G G H Z}\right\rangle=\cos \theta|000\rangle+\sin \theta|111\rangle
$$

gives rise to the Svetlichny family of quantum correlations, defined by:

$$
P_{S v F}^{\mu}=\frac{2+(-1)^{a \oplus b \oplus c \oplus x y \oplus x z \oplus y z \sqrt{2} \mu}}{16} ; \quad 0<\mu \leq 1
$$

for suitable choice of projective measurements. The above box violates the Svetlichny inequality (given in Eq. (13)) for $\mu>\frac{1}{\sqrt{2}}$, and for $\mu \leq \frac{1}{\sqrt{2}}$ it is fully local (i.e., 3-local) as in this range the correlation does not violate any Bell inequality.

The canonical decomposition of the Svetlichny family (26) is the "noisy Svetlichny-box"

$$
P_{S v F}^{\mu}=p_{S v} P_{S v}^{0000}+\left(1-p_{S v}\right) P_{N} ; \quad 0<p_{S v} \leq 1,
$$

with $p_{S v}=\mu / \sqrt{2}$, which is a special case of Eq. (18). Here, $P_{N}$ is the maximally mixed box, i.e., $P_{N}(a b c \mid x y z)=1 / 8$ for all $x, y, z, a, b, c$. That Eq. (27) is indeed canonical decomposition is proved in detail in [26]. Briefly, the proof makes use of non-trivial symmetry properties of the extreme boxes of the polytope $\mathcal{R}$, such as for example that every Svetlichny box (say, $P_{S v}^{0000}$ ) has a "complement" (here: $P_{S v}^{0001}$ ) such that their uniform mixture yields the maximally mixed box, i.e., $P_{N}=\frac{1}{2}\left(P_{S v}^{0000}+P_{S v}^{0001}\right)$. Note that the Svetlichny family $(26)$ has $\mathcal{G}=4 \sqrt{2} \mu$ and the Svetlichny-local box $P_{N}$ in the decomposition (27) of this family has $\mathcal{G}=0$, indicating that this decomposition is indeed canonical. This implies that the Svetlichny strength of the Svetlichny family can be calculated from $\mathcal{G}$ and is given by $\mathcal{G}\left(P_{S v F}^{\mu}\right) / 8=\frac{\mu}{\sqrt{2}}$ as stated above.

Therefore, the Svetlichny-box fraction in Eq. (27) can be read off as the Svetlichny strength of the Svetlichny family. Since the Svetlichny family has nonzero Svetlichny strength for any $\mu>0$, the quantum simulation of these correlations by using a threequbit system necessarily requires genuine quantumness in the state [26]. In this light, the Svetlichny strength $p_{S v}$ satisfies the relation $p_{S v}=\frac{\sqrt{\tau_{3}}}{\sqrt{2}}$, where $\tau_{3}=\sin ^{2} 2 \theta$ is the 
three-tangle [38] of $\left|\psi_{G G H Z}\right\rangle$, when the Svetlichny family is simulated by $\left|\psi_{G G H Z}\right\rangle$ for the noncommuting projective measurements corresponding to the operators: $A_{0}=\sigma_{x}$, $A_{1}=\sigma_{y}, B_{0}=\left(\sigma_{x}-\sigma_{y}\right) / \sqrt{2}, B_{1}=\left(\sigma_{x}+\sigma_{y}\right) / \sqrt{2}, C_{0}=\sigma_{x}$ and $C_{1}=\sigma_{y}$.

As noted above, for $0<\mu \leq \frac{1}{\sqrt{2}}$ the fully local Svetlichny family can be decomposed as a convex mixture of the 3-local deterministic boxes. In this range, the fully local Svetlichny family can be decomposed in the following 2-local form across the bipartition $(A \mid B C)$ :

$$
P_{S v F}^{\mu}=\sum_{\lambda=0}^{3} p_{\lambda} P_{\lambda}^{S v}(a \mid x) P_{\lambda}^{S v}(b c \mid y z),
$$

where $P_{\lambda}^{S v}(a \mid x)$ are different deterministic distributions and $P_{\lambda}^{S v}(b c \mid y z)$ are local boxes (see Appendix A for the derivation of the above decomposition). For the fully local Svetlichny family $\left(0<\mu \leq \frac{1}{\sqrt{2}}\right)$, the decomposition (28) defines a classical simulation protocol where Alice shares hidden variable $\lambda_{A-B C}$ of dimension 4 with Bob-Charlie as in Fig. 1.

Theorem 1. The fully local Svetlichny family $P_{S v F}^{\mu}\left(0<\mu \leq \frac{1}{\sqrt{2}}\right)$ is genuinely tripartite superlocal.

Proof. Let us try to reproduce the fully local Svetlichny family $P_{S v F}^{\mu}\left(0<\mu \leq \frac{1}{\sqrt{2}}\right)$ in the scenario as in Fig. 1 where Alice preshares the hidden variable $\lambda_{A-B C}$ of dimension 2 with Bob-Charlie. Before proceeding, we want to mention that in case of Svetlichny family, all the marginal probability distributions of Alice, Bob and Charlie are maximally mixed:

$$
P_{S v F}(a \mid x)=P_{S v F}(b \mid y)=P_{S v F}(c \mid z)=\frac{1}{2} \forall a, b, c, x, y, z .
$$

Let us now try to check whether the fully local Svetlichny family $P_{S v F}^{\mu}\left(0<\mu \leq \frac{1}{\sqrt{2}}\right)$ can be decomposed in the following form:

$$
P_{S v F}^{\mu}=\sum_{\lambda=0}^{1} p_{\lambda} P_{\lambda}^{S v}(a \mid x) P_{\lambda}^{S v}(b c \mid y z)
$$

where $p_{0}=x_{0}, p_{1}=x_{1}\left(0<x_{0}<1,0<x_{1}<1, x_{0}+x_{1}=1\right)$ and $P_{\lambda}^{S v}(b c \mid y z)$ are local boxes. Let us assume that Alice's strategy to be deterministic one, i.e., each of the two probability distributions $P_{0}^{S v}(a \mid x)$ and $P_{1}^{S v}(a \mid x)$ in the above decomposition belongs to any one among $P_{D}^{00}, P_{D}^{01}, P_{D}^{10}$ and $P_{D}^{11}$. In order to satisfy the marginal probabilities for Alice $P_{S v F}(a \mid x)$, the only two possible choices of $P_{0}^{S v}(a \mid x)$ and $P_{1}^{S v}(a \mid x)$ are:

(i) $P_{D}^{00}$ and $P_{D}^{01}$ with $x_{0}=x_{1}=\frac{1}{2}$

(ii) $P_{D}^{10}$ and $P_{D}^{11}$ with $x_{0}=x_{1}=\frac{1}{2}$.

Now, it can be easily checked that none of these two possible choices will satisfy all the tripartite joint probability distributions $P_{S v F}^{\mu}$ simultaneously (for detailed calculations, see Appendix B). It is, therefore, impossible to reproduce the fully local box $P_{S v F}^{\mu}\left(0<\mu \leq \frac{1}{\sqrt{2}}\right)$ in the scenario as in Fig. 1 where Alice preshares the hidden variable $\lambda_{A-B C}$ of dimension 2 with Bob-Charlie and uses deterministic strategy for each $\lambda_{A-B C}$. 
The fully local box $P_{S v F}^{\mu}\left(0<\mu \leq \frac{1}{\sqrt{2}}\right)$ cannot be reproduced by a classical simulation model as in Eq. (30) with hidden variable $\lambda_{A-B C}$ of dimension 2 even if Alice uses nondeterministic strategy for each $\lambda_{A-B C}$. To see this, we note that from any decomposition of the fully local box in terms of fully deterministic boxes (4), one may derive a classical simulation protocol as in Fig. 1 with different deterministic distributions at Alice's side. Any such classical simulation protocol does not require Alice to preshare the hidden variable $\lambda_{A-B C}$ of dimension more than 4 since there are only 4 possible different deterministic distributions given by Eq. (5) at Alice's side. Hence, a classical simulation model with hidden variable $\lambda_{A-B C}$ of dimension 2 of the fully local box $P_{S v F}^{\mu}\left(0<\mu \leq \frac{1}{\sqrt{2}}\right)$ can be achieved by constructing a classical simulation model of the fully local box $P_{S v F}^{\mu}$ with hidden variable $\lambda_{A-B C}$ of dimension 3 or 4 with different deterministic distributions at Alice's side followed by taking equal joint probability distributions at Bob-Charlie's side as common and making the corresponding distributions at Alice's side nondeterministic.

Let us now try check whether the fully local noisy Svetlichny-box $P_{S v F}^{\mu}(0<\mu \leq$ $\left.\frac{1}{\sqrt{2}}\right)$ can be simulated by a classical simulation model in the scenario as in Fig. 1 where Alice shares the hidden variable of dimension $d_{\lambda_{A-B C}}=3$ and uses different deterministic strategy at each $\lambda_{A-B C}$. In this case, we assume that the box can be decomposed in the following way:

$$
P_{S v F}^{\mu}=\sum_{\lambda=0}^{2} p_{\lambda} P_{\lambda}^{S v}(a \mid x) P_{\lambda}^{S v}(b c \mid y z) .
$$

Here, $p_{0}=x_{0}, p_{1}=x_{1}, p_{2}=x_{2}\left(0<x_{0}<1,0<x_{1}<1,0<x_{2}<1, x_{0}+x_{1}+x_{2}=1\right)$ and $P_{\lambda}^{S v}(a \mid x)$ are deterministic distributions and $P_{\lambda}^{S v}(b c \mid y z)$ are local boxes. Since Alice's distributions are deterministic, the three probability distributions $P_{0}^{S v}(a \mid x)$, $P_{1}^{S v}(a \mid x)$ and $P_{2}^{S v}(a \mid x)$ must be equal to any three among $P_{D}^{00}, P_{D}^{01}, P_{D}^{10}$ and $P_{D}^{11}$. But any such combination will not satisfy the marginal probabilities $P_{S v F}(a \mid x)$ for Alice. This implies that the fully local box $P_{S v F}^{\mu}\left(0<\mu \leq \frac{1}{\sqrt{2}}\right)$ cannot be reproduced in any classical simulation protocol with different deterministic distributions $P_{\lambda}^{S v}(a \mid x)$ at Alice's side, where Alice preshares the hidden variable $\lambda_{A-B C}$ of dimension 3 with Bob-Charlie.

Therefore, in the classical simulation model for the fully local Svetlichny family in the scenario as in Fig. 1 where Alice uses deterministic strategies, Alice has to share the hidden variable of dimension $d_{\lambda_{A-B C}}=4$.

Suppose the fully local Svetlichny family $P_{S v F}^{\mu}\left(0<\mu \leq \frac{1}{\sqrt{2}}\right)$ can be reproduced by the following classical simulation model:

$$
P_{S v F}^{\mu}=\sum_{\lambda=0}^{3} p_{\lambda} P_{\lambda}^{S v}(a \mid x) P_{\lambda}^{S v}(b c \mid y z),
$$

where $P_{\lambda}^{S v}(a \mid x)$ are different deterministic distributions and either any three of the four joint probability distributions $P_{\lambda}^{S v}(b c \mid y z)$ are equal to each other, or there exists two sets each containing two equal joint probability distributions $P_{\lambda}^{S v}(b c \mid y z) ; 0<p_{\lambda}<1$ for $\lambda=0,1,2,3 ; \sum_{\lambda=0}^{3} p_{\lambda}=1$. Then taking equal joint probability distributions $P_{\lambda}^{S v}(b c \mid y z)$ at Bob-Charlie's side as common and making corresponding distribution at Alice's side non-deterministic will reduce the dimension of the hidden variable 
$\lambda_{A-B C}$ from 4 to 2. For example, let us consider

$$
P_{0}^{S v}(b c \mid y z)=P_{1}^{S v}(b c \mid y z)=P_{2}^{S v}(b c \mid y z) .
$$

Now in order to satisfy Alice's marginal given by Eq. (29), one must take $p_{0}=p_{1}=$ $p_{2}=p_{3}=\frac{1}{4}$. Hence, the decomposition (32) can be written as,

$$
P_{S v F}^{\mu}=q_{0} \mathbb{P}_{0}^{S v}(a \mid x) P_{0}^{S v}(b c \mid y z)+p_{3} P_{3}^{S v}(a \mid x) P_{3}^{S v}(b c \mid y z),
$$

where $\mathbb{P}_{0}^{S v}(a \mid x)=\frac{P_{0}^{S v}(a \mid x)+P_{1}^{S v}(a \mid x)+P_{2}^{S v}(a \mid x)}{3}$, which is a non-deterministic distribution at Alice's side, and $q_{0}=\frac{3}{4}$. The decomposition (34) represents a classical simulation protocol of the fully local Svetlichny family $P_{S v F}^{\mu}\left(0<\mu \leq \frac{1}{\sqrt{2}}\right)$ with different deterministic/non-deterministic distributions at Alice's side, where Alice shares hidden variable $\lambda_{A-B C}$ of dimension 2 with Bob-Charlie. Now in this protocol, considering arbitrary joint probability distributions $P_{\lambda}^{S v}(b c \mid y z)$ at Bob-Charlie's side (without considering any constraint as in the case presented in Appendix B), it can be checked that all the tripartite distributions of $P_{S v F}^{\mu}$ are not reproduced simultaneously.

There are the following other cases in which the dimension of the hidden variable $\lambda_{A-B C}$ can be reduced from 4 to 2 in the classical simulation model as in Eq. (32):

$$
\begin{aligned}
& P_{0}^{S v}(b c \mid y z)=P_{2}^{S v}(b c \mid y z)=P_{3}^{S v}(b c \mid y z) ; \\
& P_{0}^{S v}(b c \mid y z)=P_{1}^{S v}(b c \mid y z)=P_{3}^{S v}(b c \mid y z) ; \\
& P_{1}^{S v}(b c \mid y z)=P_{2}^{S v}(b c \mid y z)=P_{3}^{S v}(b c \mid y z) \\
& P_{0}^{S v}(b c \mid y z)=P_{1}^{S v}(b c \mid y z) \text { as well as } P_{2}^{S v}(b c \mid y z)=P_{3}^{S v}(b c \mid y z) ; \\
& P_{0}^{S v}(b c \mid y z)=P_{2}^{S v}(b c \mid y z) \text { as well as } P_{1}^{S v}(b c \mid y z)=P_{3}^{S v}(b c \mid y z) ; \\
& P_{0}^{S v}(b c \mid y z)=P_{3}^{S v}(b c \mid y z) \text { as well as } P_{1}^{S v}(b c \mid y z)=P_{2}^{S v}(b c \mid y z) .
\end{aligned}
$$

Now in any of these possible cases, considering arbitrary joint probability distributions $P_{\lambda}^{S v}(b c \mid y z)$ at Bob-Charlie's side (without considering any constraint), it can be checked that all the tripartite distribution $P_{S v F}^{\mu}$ are not reproduced simultaneously. Hence, this also holds when the boxes $P_{\lambda}^{S v}(b c \mid y z)$ satisfy NS principle as well as locality condition.

Hence, one can conclude that it is impossible to reduce the dimension from 4 to 2 in the classical simulation protocol of the fully local Svetlichny family $P_{S v F}^{\mu}$ $\left(0<\mu \leq \frac{1}{\sqrt{2}}\right)$ in the scenario as in Fig. 1 .

It is, therefore, impossible to reproduce the fully local box $P_{S v F}^{\mu}(0<\mu \leq$ $\left.\frac{1}{\sqrt{2}}\right)$ with deterministic/non-deterministic distributions at Alice's side, where Alice preshares the hidden variable $\lambda_{A-B C}$ of dimension 2 with Bob-Charlie in the scenario as in Fig. 1.

It can be checked that the fully local Svetlichny box $P_{S v F}^{\mu}\left(0<\mu \leq \frac{1}{\sqrt{2}}\right)$ is non-product. It is, therefore, impossible to reproduce the fully local box $P_{S v F}^{\mu}$ $\left(0<\mu \leq \frac{1}{\sqrt{2}}\right)$ in the scenario where Alice preshares the hidden variable $\lambda_{A-B C}$ of dimension 1 with Bob-Charlie.

Hence, the dimension of the hidden variable $\lambda_{A-B C}$, which Alice preshares with Bob-Charlie to reproduce the fully local box $P_{S v F}^{\mu}\left(0<\mu \leq \frac{1}{\sqrt{2}}\right)$, must be greater than 2. Therefore, the fully local Svetlichny box $P_{S v F}^{\mu}\left(0<\mu \leq \frac{1}{\sqrt{2}}\right)$ has 2-local form across the bipartite cut $(A \mid B C)$ and is not 2-sublocal across that bipartite cut. Hence, the fully local Svetlichny box $P_{S v F}^{\mu}\left(0<\mu \leq \frac{1}{\sqrt{2}}\right)$ is superlocal across that bipartite cut. 
It can be checked that a similar argument holds across the remaining two bipartite cuts $(C \mid A B)$ and $(B \mid C A)$, i.e., this box is superlocal across all three possible bipartite cuts and, hence, must be genuinely tripartite superlocal.

Since by definition, fully local correlations, which are genuinely superlocal, must be absolutely superlocal as well, it follows from Theorem 1 that the fully local Svetlichny box $P_{S v F}^{\mu}\left(0<\mu \leq \frac{1}{\sqrt{2}}\right)$ is absolutely superlocal also.

\subsection{Noisy Mermin-box}

We are now interested in quantum correlations that belong to the Mermin family defined as

$$
P_{M F}^{\nu}=\frac{1+(-1)^{a \oplus b \oplus c \oplus x y \oplus x z \oplus y z} \delta_{x \oplus y \oplus 1, z} \nu}{8} ; \quad 0<\nu \leq 1 .
$$

For $\nu \leq \frac{1}{2}$, the above box is fully local as in this range the correlation does not violate any Bell inequality. The above box is absolutely nonlocal, but 2-local for $\nu>\frac{1}{2}$ as it violates the Mermin inequality (given in Eq. (15)) for $\nu>\frac{1}{2}$, but not any of the Svetlichny inequalities. Thus, it isn't obvious that this correlation would be genuinely superlocal, yet this is what will be established below.

The Mermin family (35) has the canonical decomposition as the noisy Merminbox

$$
P_{M F}^{\nu}=p_{M} P_{M}+\left(1-p_{M}\right) P_{N} ; \quad 0<p_{M} \leq 1,
$$

with $p_{M}=\nu$, which is a special case of Eq. (20). Here, the Mermin-box $P_{M}=\frac{1}{2}\left(P_{S v}^{0000}+P_{S v}^{1110}\right)$. That Eq. (36) is indeed the canonical decomposition for Eq. (35) can be shown, as with the case of the noisy Svetlichny box, by making use of the non-trivial symmetry properties of the extremal boxes of the polytope $\mathcal{R}$, such as the fact that any given Mermin box (say, $P_{M}$ ) has a complement such that their uniform mixture yields the white noise, i.e., $P_{N}=\frac{1}{2} P_{M}+\frac{1}{2} P_{M}^{\prime}$, where $P_{M}^{\prime}=\frac{1}{2}\left(P_{S v}^{0001}+P_{S v}^{1111}\right)[26]$. Therefore, the Mermin-box fraction in Eq. (36) indeed gives Mermin strength [26] of the Mermin family. Since the Mermin family has a decomposition as in Eq. (20), its Mermin strength can be calculated from $\mathcal{Q}$ and is given by $\mathcal{Q}\left(P_{M F}^{\nu}\right) / 4=\nu$ as stated above.

For any $\nu>0$, the quantum simulation of the Mermin family by using a threequbit system necessarily requires genuine quantumness in the state, even if it is fully local or 2-local. This is due to the fact that the Mermin family has nonzero Mermin strength for any $\nu>0[26]$. Note that the GGHZ state, $\left|\psi_{G G H Z}\right\rangle$ (given by Eq. (25)), gives rise to the Mermin family with Mermin strength $\nu=\sqrt{\tau_{3}}$ for the noncommuting projective measurements corresponding to the operators: $A_{0}=\sigma_{x}, A_{1}=\sigma_{y}, B_{0}=\sigma_{x}$, $B_{1}=\sigma_{y}, C_{0}=\sigma_{x}$ and $C_{1}=\sigma_{y}$ that demonstrates the GHZ paradox.

As noted above, for $0<\nu \leq \frac{1}{2}$ the fully local noisy Mermin box can be decomposed in a convex mixture of the 3-local deterministic boxes. In this range, the fully local Mermin family can be decomposed in the following 2-local form across the bipartition $(A \mid B C)$ :

$$
P_{M F}^{\nu}=\sum_{\lambda=0}^{3} r_{\lambda} P_{\lambda}^{M}(a \mid x) P_{\lambda}^{M}(b c \mid y z)
$$


where $P_{\lambda}^{M}(a \mid x)$ are different deterministic distributions and $P_{\lambda}^{M}(b c \mid y z)$ are local boxes (see Appendix $\mathrm{C}$ for the derivation of the above decomposition). For the fully local noisy Mermin-box $\left(0<\nu \leq \frac{1}{2}\right)$, the decomposition (37) defines a classical simulation protocol where Alice shares hidden variable $\lambda_{A-B C}$ of dimension 4 as in Fig. 1.

Theorem 2. The fully local noisy Mermin box $P_{M F}^{\nu}\left(0<\nu \leq \frac{1}{2}\right)$ is genuinely tripartite superlocal.

Proof. In case of noisy Mermin-box also, all the marginal probability distributions of Alice, Bob and Charlie are maximally mixed:

$$
P_{M F}(a \mid x)=P_{M F}(b \mid y)=P_{M F}(c \mid z)=\frac{1}{2} \forall a, b, c, x, y, z .
$$

Let us try to construct a classical simulation protocol for the fully local Mermin family $P_{M F}^{\nu}\left(0<\nu \leq \frac{1}{2}\right)$ with different deterministic distributions $P_{\lambda}^{M}(a \mid x)$ at Alice's side, where Alice shares hidden variable $\lambda_{A-B C}$ of dimension 2 with Bob-Charlie. In this case, the fully local Mermin family $\left(0<\nu \leq \frac{1}{2}\right)$ can be decomposed in the following way:

$$
P_{M F}^{\nu}=\sum_{\lambda=0}^{1} r_{\lambda} P_{\lambda}^{M}(a \mid x) P_{\lambda}^{M}(b c \mid y z) .
$$

Here, $r_{0}=a_{0}, r_{1}=a_{1}\left(0<a_{0}<1,0<a_{1}<1, a_{0}+a_{1}=1\right)$. Since Alice's distributions are deterministic, the two probability distributions $P_{0}^{M}(a \mid x)$ and $P_{1}^{M}(a \mid x)$ must be equal to any two among $P_{D}^{00}, P_{D}^{01}, P_{D}^{10}$ and $P_{D}^{11}$. In order to satisfy the marginal probabilities for Alice $P_{M F}(a \mid x)$, the only two possible choices of $P_{0}^{M}(a \mid x)$ and $P_{1}^{M}(a \mid x)$ are:

1) $P_{D}^{00}$ and $P_{D}^{01}$ with $a_{0}=a_{1}=\frac{1}{2}$

2) $P_{D}^{10}$ and $P_{D}^{11}$ with $a_{0}=a_{1}=\frac{1}{2}$.

Now, in a similar process as adopted in case of Svetlichny family, it can be easily checked that none of these two possible choices will satisfy all the tripartite joint probability distributions $P_{M F}^{\nu}$ simultaneously.

Since Alice's marginal distributions are maximally mixed, it can be shown in a similar way as presented in case of noisy Svetlichny box that there does not exist a classical simulation model for the fully local Mermin family in the scenario as in Fig. 1 where Alice shares the hidden variable of dimension $d_{\lambda_{A-B C}}=3$ and uses different deterministic strategies at each $\lambda_{A-B C}$.

Thus, in the classical simulation model for the fully local Mermin family in the scenario as in Fig. 1 where Alice uses different deterministic strategies, Alice has to share the hidden variable of dimension $d_{\lambda_{A-B C}}=4$. Let us now try to check whether there exists such a classical simulation model for the fully local noisy Mermin box where $d_{\lambda_{A-B C}}$ can be reduced from 4 to 2 by allowing non-deterministic strategies on Alice's side. That is we try to construct the following classical simulation protocol for the fully local noisy Mermin-box $\left(0<\nu \leq \frac{1}{2}\right)$ with different deterministic distributions $P_{\lambda}^{M}(a \mid x)$ at Alice's side, where Alice shares hidden variable $\lambda_{A-B C}$ of dimension 4 with Bob-Charlie in the scenario as in Fig. 1:

$$
P_{M F}^{\nu}=\sum_{\lambda=0}^{3} p_{\lambda} P_{\lambda}^{M}(a \mid x) P_{\lambda}^{M}(b c \mid y z),
$$


where either any three of the four joint probability distributions $P_{\lambda}^{S M}(b c \mid y z)$ are equal to each other, or there exists two sets each containing two equal joint probability distributions $P_{\lambda}^{M}(b c \mid y z) ; 0<p_{\lambda}<1$ for $\lambda=0,1,2,3 ; \sum_{\lambda=0}^{3} p_{\lambda}=1$. Then, as described earlier in the case of noisy Svetlichny-box, taking equal joint probability distributions $P_{\lambda}^{M}(b c \mid y z)$ at Bob-Charlie's side as common and making corresponding distribution at Alice's side non-deterministic will reduce the dimension of the hidden variable $\lambda_{A-B C}$ from 4 to 2 . Now following the similar procedure adopted in case of noisy Svetlichny box, one can show that it is impossible to reduce the dimension from 4 to 2 in the classical simulation protocol of the fully local noisy Mermin-box $\left(0<\nu \leq \frac{1}{2}\right)$ in the scenario as in Fig. 1.

It is, therefore, impossible to reproduce the fully local Mermin family $P_{M F}^{\nu}$ $\left(0<\nu \leq \frac{1}{2}\right)$ in any classical simulation protocol with deterministic/non-deterministic distributions $P_{\lambda}^{M}(a \mid x)$ at Alice's side, where Alice preshare the hidden variable $\lambda_{A-B C}$ of dimension 2 with Bob-Charlie in the scenario as in Fig. 1 .

It can be checked that the fully local Mermin family $P_{M F}^{\nu}\left(0<\nu \leq \frac{1}{2}\right)$ is nonproduct. It is, therefore, impossible to reproduce the fully local Mermin family $P_{M F}^{\nu}$ $\left(0<\nu \leq \frac{1}{2}\right)$ in the scenario where Alice preshares the hidden variable $\lambda_{A-B C}$ of dimension 1 with Bob-Charlie.

Hence, the dimension of the hidden variable $\lambda_{A-B C}$, which Alice preshares with Bob-Charlie to reproduce the fully local Mermin family $P_{M F}^{\nu}\left(0<\nu \leq \frac{1}{2}\right)$, must be greater than 2. Therefore, the fully local Mermin family $P_{M F}^{\nu}\left(0<\nu \leq \frac{1}{2}\right)$ has 2local form across the bipartite cut $(A \mid B C)$ and is not 2-sublocal across that bipartite cut. Hence, the fully local Mermin family $P_{M F}^{\nu}\left(0<\nu \leq \frac{1}{2}\right)$ is superlocal across the bipartite cut $(A \mid B C)$.

It can be checked that a similar argument holds across the remaining two bipartite cuts $(C \mid A B)$ and $(B \mid C A)$, i.e., this box is superlocal across all three possible bipartite cuts and, hence, must be genuinely tripartite superlocal.

Since the fully local noisy Mermin box $P_{M F}^{\nu}\left(0<\nu \leq \frac{1}{2}\right)$ is genuinely superlocal, it is absolutely superlocal as well.

Now, as noted before, for $0<\nu \leq 1$ the 2-local Mermin family can be decomposed in a convex mixture of the local vertices and 2-local vertices. In this range, the Mermin family can be decomposed in the following 2-local form across the bipartition $(A \mid B C)$ :

$$
P_{M F}^{0<\nu<1}=\sum_{\lambda=0}^{3} r_{\lambda} P_{\lambda}^{M}(a \mid x) P_{\lambda}^{M}(b c \mid y z),
$$

where $P_{\lambda}^{M}(a \mid x)$ are different deterministic distributions and $P_{\lambda}^{M}(b c \mid y z)$ are NS boxes (see Appendix D for the derivation of the above decomposition). For the 2-local noisy Mermin-box $(0<\nu \leq 1)$, the decomposition (41) defines a classical simulation protocol as in Fig. 2 where Alice shares hidden variable $\lambda_{A-B C}$ of dimension 4.

Since the fully local noisy Mermin box is a special case of the 2-local noisy Mermin box and the proof of Theorem 2 is independent of the locality condition of the bipartite distributions $P_{\lambda}^{M}(b c \mid y z)$ at Bob-Charlie's side, the proof is also valid when the bipartite distributions $P_{\lambda}^{M}(b c \mid y z)$ at Bob-Charlie's side are NS (local or nonlocal) boxes. Hence, it is not difficult to see that the proof of the Theorem 2 can be straightforwardly adopted to obtain the following result:

Theorem 3. The 2-local noisy Mermin box $P_{M F}^{\nu}(0<\nu \leq 1)$ is genuinely tripartite superlocal. 


\section{Connection between genuine super-locality and genuine nonclassicality}

In Ref. [22], Giorgi et al defined genuine tripartite quantum discord to quantify the quantum part of genuine tripartite correlations in a tripartite quantum state. As the name suggests, this kind of quantifier also captures genuine quantumness of separable states. Genuine tripartite quantum discord defined in Ref. [24] goes to zero iff there exists a bipartite cut of the tripartite system such that no quantum correlation exists between the two parts. It is known that a bipartite quantum state has no quantum correlation as quantified by Alice to Bob quantum discord iff it can be written in the classical-quantum state form [39]. We define tripartite classical-quantum states as follows.

Definition. A fully separable tripartite state has a classical-quantum state form across the bipartite cut $(A \mid B C)$ if it can be decomposed as

$$
\rho_{C Q}^{A \mid B C}=\sum_{i} p_{i}|i\rangle^{A}\langle i| \otimes \rho_{i}^{B} \otimes \rho_{i}^{C},
$$

where $\left\{|i\rangle^{A}\right\}$ is some orthonormal basis of Alice's Hilbert space $\mathcal{H}_{A}$.

Note that the classical-quantum states as defined above do not have nonzero genuine quantum discord since Alice's subsystem is always classically correlated with Bob and Charlie's subsystem. We characterize a (the fully separable) state as having genuine quantumness, if it cannot be written in the classical-quantum state in any bipartite cut as in Eq. (42).

The Svetlichny family and Mermin family violate a three-qubit biseparability inequality for $\mu>1 / 2$ and $\nu>1 / 2$ [40]. Therefore, in that range these two families certify genuine three-qubit entanglement in a semi-device-independent way as well $[40,41]$. For $\mu \leq 1 / 2$ and $\nu \leq 1 / 2$, the Svetlichny and Mermin families can also be reproduced by separable three-qubit states since they do not violate any biseparability inequality in this range.

However, for $\mu, \nu \in\left(0, \frac{1}{2}\right]$, the simulation of the Svetlichny family and Mermin family by using three-qubit separable states serves to witness genuine quantumness in the form of genuine quantum discord as they have nonzero Svetlichny strength and nonzero Mermin strength, respectively [26]. This observation prompts us to make the following observation.

Observation 1. Genuine quantumness (i.e., nonzero discord across any bipartite cut) of any correlation $P$ is necessary for genuine superlocality.

Proof. Consider tripartite boxes arising from three-qubit classical-quantum states which have the form as given in Eq. (42) with $i=0,1$. In the Bell scenario that we have considered, for Alice measuring in basis $\{|i\rangle\}$, it is clear that the resulting box can be simulated by a probabilistic strategy using dimension $d_{\lambda_{A}}=2$ on Alice's side. This observation holds even when Alice measures in any other basis (except that her random number generator will be possibly be more randomized). This implies that for any three-qubit state which do not have genuine quantumness, there exists a bipartite cut in which it is not superlocal. Therefore, genuine quantum discord is necessary for implying genuine superlocality. 


\section{Genuine multipartite nonclassicality}

Generalizing the definitions presented in Section 2, an $n$-partite correlation is said to be fully local or $n$-local if the box has a decomposition of the form:

$$
\begin{aligned}
& P\left(a_{1}, a_{2}, \cdots, a_{n} \mid x_{1}, x_{2}, \cdots, x_{n}\right)= \\
& \sum_{\lambda} p_{\lambda} P_{\lambda}\left(a_{1} \mid x_{1}\right) P_{\lambda}\left(a_{2} \mid x_{2}\right) \cdots P_{\lambda}\left(a_{n} \mid x_{n}\right),
\end{aligned}
$$

where $\sum_{\lambda} p_{\lambda}=1$, and $x_{1}, x_{2}, \cdots, x_{n}$ denote the inputs (measurement choices) and $a_{1}, a_{2}, \cdots, a_{n}$ denote the outputs (measurement outcomes) of the parties $q_{1}, q_{2}, \ldots, q_{n}$ respectively. An $n$-partite box that is not $n$-local is said to have "absolute nonlocality".

A $n$-partite correlation is said to be $k$-local if it can be decomposed as a convex combination of $k$-partitions such that these $k$ parts (defined by each $k$-partition) are locally correlated with each other. For example, a 4-partite correlation is 3-local if it can be decomposed as a convex combination of tripartitions such as $\left(q_{1}\left|q_{2} q_{3}\right| q_{4}\right)$ such that these parts are locally correlated each other. However, $q_{2} q_{3}$ may be nonlocal in itself. Similarly, a 4-partite correlation is 2-local if it can be decomposed as a convex combination of probability distributions over bipartitions such that in each bipartition, the two parts are locally correlated, though within a part, even nonlocality may hold. Any $k$-local correlation is also $k^{\prime}$-local where $k^{\prime}<k$. Thus, a 4-local correlation is also 3 -local and a 3-local correlation is also 2-local. But the converse is not true.

Therefore, the weakest form of locality is 2-locality, and the strongest form of nonlocality for an $n$-partite system is that which is not 2-local. This is called genuine $n$-partite nonlocality, for which all bipartitions are nonlocal.

An $n$-partite system is $n$-sublocal (or, fully sublocal) if each of the $n$ particles are locally correlated, with the shared classical randomness dimension being less than or equal to the smallest local Hilbert space dimension among all the $n$ particles, in analogy with Eq. (23):

Definition. Suppose we have an $n$-partite quantum state in $\mathbb{C}^{d_{s_{1}}} \otimes \mathbb{C}^{d_{s_{2}}} \otimes \cdots \otimes \mathbb{C}^{d_{s_{n}}}$ and measurements which produce a fully local $n$-partite box $P\left(a_{1} a_{2} \cdots a_{n} \mid x_{1} x_{2} \cdots x_{n}\right)$. The correlation $P$ is $n$-sublocal (or, fully sublocal) precisely if there exists a decomposition such that

$$
\begin{aligned}
& P\left(a_{1} a_{2} \cdots a_{n} \mid x_{1} x_{2} \cdots x_{n}\right)= \\
& \quad \sum_{\lambda=0}^{d_{\lambda}-1} p_{\lambda} P_{\lambda}\left(a_{1} \mid x_{1}\right) P_{\lambda}\left(a_{2} \mid x_{2}\right) \cdots P_{\lambda}\left(a_{n} \mid x_{n}\right),
\end{aligned}
$$

with $d_{\lambda} \leq d$, and $d=\min \left\{d_{s_{1}}, d_{s_{2}}, \cdots, d_{s_{n}}\right\}$, where $d_{s_{j}}$ is the local Hilbert space dimension of the $j$ th particle. The fully local correlation $P$ is absolutely superlocal if it is not $n$-sublocal. In other words, absolute $n$-partite superlocality holds iff there is no $n$-sublocal decomposition (44) of the given fully local box. Here $\sum_{\lambda=0}^{d_{\lambda}-1} p_{\lambda}=1$.

A $n$-partite correlation $P$ is said to be " $k$-sublocal across a particular $k$-partition" if these $k$ parts are sublocally correlated with each other. For example, a 4-partite correlation is 3 -sublocal across the tripartite cut $\left(q_{1}\left|q_{2} q_{3}\right| q_{4}\right)$ if these three parts are sublocally correlated with each other. Note that the part $\left(q_{2} q_{3}\right)$ may be sublocal or superlocal or even nonlocal. 
Thus, a correlation which is 4-sublocal across some 4-partition $Q^{(|| l)}$, is also 3sublocal across any tripartition $Q^{(\|)}$obtained by merging any two partitions of $Q^{(\| l)}$, and also 2-sublocal across any bipartition $Q^{(\mid)}$obtained by merging any two partitions of $Q^{(\|)}$.

In general, any correlation, which is $k$-sublocal across some $k$-partition, is also $k^{\prime}$-sublocal across some $k^{\prime}$-partition where $k^{\prime}<k$, where the $k^{\prime}$-partition has been obtained by merging parts of the $k$-partition. Therefore, the weakest form of sublocality is 2-sublocality. An n-partite correlation $P$ that isn't sublocal - or equivalently, is superlocal-across every bipartition, is genuinely n-partite superlocal. Note that, because of the non-convexity associated with sublocal sets, a convex combination of 2-sublocal correlations needn't itself be 2 -sublocal.

In line with our definition for superlocality for a multipartite system, we may define $n$-concord as the absence of quantum discord across all cuts splitting all $n$ particles, i.e., $\left(q_{1}\left|q_{2}\right| \cdots \mid q_{n}\right)$. For example, the system $q_{1} q_{2} q_{3} q_{4}$ has 4-concord if there is no quantum discord across each of the cuts $q_{1}\left|q_{2}\right| q_{3} \mid q_{4}$. Absolute $n$-partite discord [42] holds when the state in question is not $n$-concordant. Genuine $n$-partite discord holds when the state in question lacks 2-concord form across all possible bipartitions [22-25]. The relation between different such measures of discord and superlocality for multipartite systems, such as that noted for the tripartite system in Section 4 , is an interesting issue meriting further studies.

\section{Conclusions}

In Ref. [26], two quantities called, Svetlichny strength and Mermin strength, have been introduced to study genuine nonclassicality of tripartite correlations. By using these two quantities, it has been demonstrated that genuine tripartite quantum discord is necessary to simulate certain fully local or 2-local tripartite correlations if the measured tripartite systems are restricted to be three-qubit states.

Genuine multipartite quantum nonlocality occurs if the multipartite correlation cannot be expressed as a convex combination of all possible bipartitions where the 2parts defined by each of these bipartitions are locally correlated with each other. Our motivation has been to perform the characterization of genuine nonclassicality of local (fully or partially local) tripartite correlations arising from the concept of superlocality, and relating this to genuine quantum discord. Thus, genuine superlocality, i.e., the occurrence of superlocality across all bipartitions, provides an operational definition of genuine nonclassicality. We have studied how genuine superlocality occurs for two families of local tripartite correlations having their nonclassicality quantified in terms of nonzero Svetlichny strength and nonzero Mermin strength, respectively.

In Ref. [10], it was demonstrated that certain bipartite separable states having quantum discord may improve the so-called random access codes (RAC), which is a class of communication problem, if the shared randomness between the two parties is limited to be finite. Recently, in Ref. [43], a family of RAC protocols have been considered in tripartite quantum networks and is associated with genuine tripartite nonlocality. Our work on characterizing genuine quantumness of certain local tripartite or multipartite correlations in the limited dimensional simulation scenario and its link with genuine quantum discord motivates the following study. It would be interesting to investigate quantum advantage for the above RAC protocol associated with tripartite or multipartite quantum networks in the presence of limited shared randomness by using tripartite or multipartite separable states with genuine 
quantum discord.

\section{Acknowledgements}

CJ thanks Manik Banik and Arup Roy for discussions. DD acknowledges the financial support from University Grants Commission (UGC), Government of India. CJ, SG and ASM acknowledge support through Project SR/S2/LOP-08/2013 of the DST, Govt. of India. 
[1] Ryszard Horodecki, Paweł Horodecki, Michał Horodecki, and Karol Horodecki. Quantum entanglement. Rev. Mod. Phys., 81:865-942, Jun 2009.

[2] Nicolas Brunner, Daniel Cavalcanti, Stefano Pironio, Valerio Scarani, and Stephanie Wehner. Bell nonlocality. Rev. Mod. Phys., 86:419-478, Apr 2014.

[3] J. S. Bell. On the einstein-podolsky-rosen paradox. Physics, 1:195, Sep 1964.

[4] Sandu Popescu and Daniel Rohrlich. Quantum nonlocality as an axiom. Found. Phys., 24(3):379-385, 1994.

[5] S Popescu. Nonlocality beyond quantum mechanics. Nat Phys, 10:264, 2014.

[6] Antonio Acín, Nicolas Gisin, and Lluis Masanes. From bell's theorem to secure quantum key distribution. Phys. Rev. Lett., 97:120405, Sep 2006.

[7] Nicolas Brunner, Daniel Cavalcanti, Alejo Salles, and Paul Skrzypczyk. Bound nonlocality and activation. Phys. Rev. Lett., 106:020402, Jan 2011.

[8] Harold Ollivier and Wojciech H. Zurek. Quantum discord: A measure of the quantumness of correlations. Phys. Rev. Lett., 88:017901, Dec 2001.

[9] Kavan Modi, Aharon Brodutch, Hugo Cable, Tomasz Paterek, and Vlatko Vedral. The classicalquantum boundary for correlations: Discord and related measures. Rev. Mod. Phys., 84:16551707, Nov 2012.

[10] Tan Kok Chuan Bobby and Tomasz Paterek. Separable states improve protocols with finite randomness. New Journal of Physics, 16(9):093063, 2014.

[11] Joseph Bowles, Flavien Hirsch, Marco Túlio Quintino, and Nicolas Brunner. Local hidden variable models for entangled quantum states using finite shared randomness. Phys. Rev. Lett., 114:120401, Mar 2015.

[12] John Matthew Donohue and Elie Wolfe. Identifying nonconvexity in the sets of limiteddimension quantum correlations. Phys. Rev. A, 92:062120, Dec 2015.

[13] K. T. Goh, J.-D. Bancal, and V. Scarani. Measurement-device-independent quantification of entanglement for given hilbert space dimension. New J. Phys., 18, 2016.

[14] C. Jebaratnam, S. Aravinda, and R. Srikanth. Nonclassicality of local bipartite correlations. Phys. Rev. A, 95:032120, Mar 2017.

[15] Marco Piani, Paweł Horodecki, and Ryszard Horodecki. No-local-broadcasting theorem for multipartite quantum correlations. Phys. Rev. Lett., 100:090502, Mar 2008.

[16] C Jebarathinam. Characterizing quantum correlations in the nonsignaling framework. PhD thesis, Indian Institute of Science Education and Research Mohali, 2016.

[17] D. M. Greenberger, M. A. Horne, and A. Zeilinger. in Bell's Theorem, Quantum Theory, and Conceptions of the Universe. Kluwer Academic Dordrecht, 1989.

[18] George Svetlichny. Distinguishing three-body from two-body nonseparability by a bell-type inequality. Phys. Rev. D, 35:3066-3069, May 1987.

[19] N. David Mermin. Extreme quantum entanglement in a superposition of macroscopically distinct states. Phys. Rev. Lett., 65:1838-1840, Oct 1990.

[20] T. Pramanik and A. S. Majumdar. Fine-grained uncertainty relation and nonlocality of tripartite systems. Phys. Rev. A, 85:024103, Feb 2012.

[21] Ansuman Dey, T. Pramanik, and A. S. Majumdar. Fine-grained uncertainty relation and biased nonlocal games in bipartite and tripartite systems. Phys. Rev. A, 87:012120, Jan 2013.

[22] Gian Luca Giorgi, Bruno Bellomo, Fernando Galve, and Roberta Zambrini. Genuine quantum and classical correlations in multipartite systems. Phys. Rev. Lett., 107:190501, Nov 2011.

[23] Jonas Maziero and Fábio M. Zimmer. Genuine multipartite system-environment correlations in decoherent dynamics. Phys. Rev. A, 86:042121, Oct 2012.

[24] Liming Zhao, Xueyuan Hu, R.-H. Yue, and Heng Fan. Genuine correlations of tripartite system. Quantum Inf. Process, 12(7):2371-2383, 2013.

[25] Andrea Beggi, Fabrizio Buscemi, and Paolo Bordone. Analytical expression of genuine tripartite quantum discord for symmetrical x-states. Quantum Inf. Process, 14(2):573-592, 2015.

[26] C. Jebaratnam. Study of nonclassicality in tripartite correlations beyond standard bell nonlocality paradigm. Quantum Studies: Mathematics and Foundations, 4(3):225-242, Sep 2017.

[27] N. David Mermin. Simple unified form for the major no-hidden-variables theorems. Phys. Rev. Lett., 65:3373-3376, Dec 1990.

[28] S. M. Roy and Virendra Singh. Tests of signal locality and einstein-bell locality for multiparticle systems. Phys. Rev. Lett., 67:2761-2764, Nov 1991.

[29] Dipankar Home and A. S. Majumdar. Incompatibility between quantum mechanics and classical realism in the "strong" macroscopic limit. Phys. Rev. A, 52:4959-4962, Dec 1995.

[30] Adán Cabello. Bell's inequality for $n$ spin-s particles. Phys. Rev. A, 65:062105, Jun 2002.

[31] Jonathan Barrett, Noah Linden, Serge Massar, Stefano Pironio, Sandu Popescu, and David 
Roberts. Nonlocal correlations as an information-theoretic resource. Phys. Rev. A, 71:022101, Feb 2005.

[32] Arthur Fine. Hidden variables, joint probability, and the bell inequalities. Phys. Rev. Lett., 48:291-295, Feb 1982.

[33] R. F. Werner and M. M. Wolf. All-multipartite bell-correlation inequalities for two dichotomic observables per site. Phys. Rev. A, 64:032112, Aug 2001.

[34] Jean-Daniel Bancal, Jonathan Barrett, Nicolas Gisin, and Stefano Pironio. Definitions of multipartite nonlocality. Phys. Rev. A, 88:014102, Jul 2013.

[35] Itamar Pitowsky and Karl Svozil. Optimal tests of quantum nonlocality. Phys. Rev. A, 64:014102, Jun 2001.

[36] Cezary liwa. Symmetries of the bell correlation inequalities. Physics Letters A, 317(34):165 $168,2003$.

[37] Stefano Pironio, Jean-Daniel Bancal, and Valerio Scarani. Extremal correlations of the tripartite no-signaling polytope. Journal of Physics A: Mathematical and Theoretical, 44(6):065303, 2011.

[38] Valerie Coffman, Joydip Kundu, and William K. Wootters. Distributed entanglement. Phys. Rev. A, 61:052306, Apr 2000.

[39] Borivoje Dakić, Vlatko Vedral, and Časlav Brukner. Necessary and sufficient condition for nonzero quantum discord. Phys. Rev. Lett., 105:190502, Nov 2010.

[40] C. Jebaratnam, Debarshi Das, Arup Roy, Amit Mukherjee, Some Sankar Bhattacharya, Bihalan Bhattacharya, Alberto Riccardi, and Debasis Sarkar. Tripartite entanglement detection through tripartite quantum steering in one-sided and two-sided device-independent scenarios. arXiv:1704.08162 [quant-ph].

[41] C. Jebaratnam. Detecting genuine multipartite entanglement in steering scenarios. Phys. Rev. A, 93:052311, May 2016.

[42] Kavan Modi, Tomasz Paterek, Wonmin Son, Vlatko Vedral, and Mark Williamson. Unified view of quantum and classical correlations. Phys. Rev. Lett., 104:080501, Feb 2010.

[43] Alley Hameedi, Debashis Saha, Piotr Mironowicz, Marcin Pawłowski, and Mohamed Bourennane. Complementarity between entanglement-assisted and quantum distributed random access code. Phys. Rev. A, 95:052345, May 2017.

[44] John F. Clauser, Michael A. Horne, Abner Shimony, and Richard A. Holt. Proposed experiment to test local hidden-variable theories. Phys. Rev. Lett., 23:880-884, Oct 1969.

[45] R. F. Werner and M. M. Wolf. Bell inequalities and entanglement. Quantum Inf. Comput., $1: 1,2001$.

[46] Debarshi Das, Bihalan Bhattacharya, Chandan Datta, Arup Roy, C. Jebaratnam, A. S. Majumdar, and R. Srikanth. Operational characterization of quantumness of unsteerable bipartite states. Phys. Rev. A, 97:062335, Jun 2018. 
Appendix A. 2-local form across the bipartition $(A \mid B C)$ for the fully local Svetlichny family $P_{S v F}^{\mu}$ in the range $0<\mu \leq \frac{1}{\sqrt{2}}$

For $0<\mu \leq \frac{1}{\sqrt{2}}$ the fully local Svetlichny family can be decomposed as a convex mixture of the 3-local deterministic boxes. In this range, we consider the following decomposition for the Svetlichny family in terms of the 3-local deterministic boxes:

$$
\begin{aligned}
P_{S v F}^{\mu} & =\frac{1}{4} P_{D}^{00}\left\{\frac{\sqrt{2} \mu}{4}\left(P_{D}^{0010}+P_{D}^{0111}+P_{D}^{1000}+P_{D}^{1101}\right)\right. \\
& \left.+\frac{1-\sqrt{2} \mu}{4}\left(P_{D}^{1000}+P_{D}^{1100}+P_{D}^{1101}+P_{D}^{1001}\right)\right\} \\
& +\frac{1}{4} P_{D}^{01}\left\{\frac{\sqrt{2} \mu}{4}\left(P_{D}^{0011}+P_{D}^{0110}+P_{D}^{1001}+P_{D}^{1100}\right)\right. \\
& \left.+\frac{1-\sqrt{2} \mu}{4}\left(P_{D}^{1000}+P_{D}^{1100}+P_{D}^{1101}+P_{D}^{1001}\right)\right\} \\
& +\frac{1}{4} P_{D}^{10}\left\{\frac{\sqrt{2} \mu}{4}\left(P_{D}^{0000}+P_{D}^{0101}+P_{D}^{1011}+P_{D}^{1110}\right)\right. \\
& \left.+\frac{1-\sqrt{2} \mu}{4}\left(P_{D}^{0000}+P_{D}^{0101}+P_{D}^{0001}+P_{D}^{0100}\right)\right\} \\
& +\frac{1}{4} P_{D}^{10}\left\{\frac{\sqrt{2} \mu}{4}\left(P_{D}^{0001}+P_{D}^{0100}+P_{D}^{1010}+P_{D}^{1111}\right)\right. \\
& \left.+\frac{1-\sqrt{2} \mu}{4}\left(P_{D}^{0000}+P_{D}^{0101}+P_{D}^{0001}+P_{D}^{0100}\right)\right\} \\
& :=\sum_{\lambda=0}^{3} p_{\lambda} P_{\lambda}^{S v}(a \mid x) P_{\lambda}^{S v}(b c \mid y z)
\end{aligned}
$$

where $p_{0}=p_{1}=p_{2}=p_{3}=\frac{1}{4}$;

$$
\begin{array}{ll}
P_{0}^{S v}(a \mid x)=P_{D}^{00}, & P_{1}^{S v}(a \mid x)=P_{D}^{01} \\
P_{2}^{S v}(a \mid x)=P_{D}^{10}, & P_{3}^{S v}(a \mid x)=P_{D}^{11}
\end{array}
$$


and

$$
\begin{aligned}
& P_{0}^{S v}(b c \mid y z)=\left\{\frac{\sqrt{2} \mu}{4}\left(P_{D}^{0010}+P_{D}^{0111}+P_{D}^{1000}+P_{D}^{1101}\right)\right. \\
& \left.+\frac{1-\sqrt{2} \mu}{4}\left(P_{D}^{1000}+P_{D}^{1100}+P_{D}^{1101}+P_{D}^{1001}\right)\right\}
\end{aligned}
$$

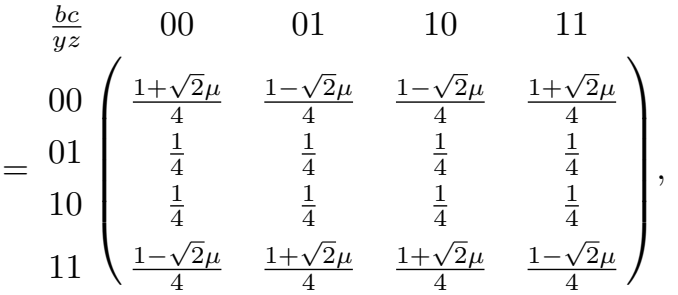

where each row and column corresponds to a fixed measurement $(y z)$ and a fixed outcome $(b c)$ respectively $\ddagger$,

$$
\begin{aligned}
& P_{1}^{S v}(b c \mid y z)=\left(\begin{array}{cccc}
\frac{1-\sqrt{2} \mu}{4} & \frac{1+\sqrt{2} \mu}{4} & \frac{1+\sqrt{2} \mu}{4} & \frac{1-\sqrt{2} \mu}{4} \\
\frac{1}{4} & \frac{1}{4} & \frac{1}{4} & \frac{1}{4} \\
\frac{1}{4} & \frac{1}{4} & \frac{1}{4} & \frac{1}{4} \\
\frac{1+\sqrt{2} \mu}{4} & \frac{1-\sqrt{2} \mu}{4} & \frac{1-\sqrt{2} \mu}{4} & \frac{1+\sqrt{2} \mu}{4}
\end{array}\right), \\
& P_{2}^{S v}(b c \mid y z)=\left(\begin{array}{cccc}
\frac{1}{4} & \frac{1}{4} & \frac{1}{4} & \frac{1}{4} \\
\frac{1+\sqrt{2} \mu}{4} & \frac{1-\sqrt{2} \mu}{4} & \frac{1-\sqrt{2} \mu}{4} & \frac{1+\sqrt{2} \mu}{4} \\
\frac{1+\sqrt{2} \mu}{4} & \frac{1-\sqrt{2} \mu}{4} & \frac{1-\sqrt{2} \mu}{4} & \frac{1+\sqrt{2} \mu}{4} \\
\frac{1}{4} & \frac{1}{4} & \frac{1}{4} & \frac{1}{4}
\end{array}\right), \\
& P_{3}^{S v}(b c \mid y z)=\left(\begin{array}{cccc}
\frac{1}{4} & \frac{1}{4} & \frac{1}{4} & \frac{1}{4} \\
\frac{1-\sqrt{2} \mu}{4} & \frac{1+\sqrt{2} \mu}{4} & \frac{1+\sqrt{2} \mu}{4} & \frac{1-\sqrt{2} \mu}{4} \\
\frac{1-\sqrt{2} \mu}{4} & \frac{1+\sqrt{2} \mu}{4} & \frac{1+\sqrt{2} \mu}{4} & \frac{1-\sqrt{2} \mu}{4} \\
\frac{1}{4} & \frac{1}{4} & \frac{1}{4} & \frac{1}{4}
\end{array}\right) .
\end{aligned}
$$

Note that, in the range $0<\mu \leq \frac{1}{\sqrt{2}}$, each of the $P_{\lambda}^{S v}(b c \mid y z)$ given above belongs to BB84 family [13] defined as

$$
P_{B B 84}(b c \mid y z)=\frac{1+(-1)^{b \oplus c \oplus y \cdot z} \delta_{y, z} V}{4},
$$

with $0<V=\sqrt{2} \mu \leq 1$, upto local reversible operations. The above family is local, as it satisfies the complete set of Bell-CHSH (Bell-Clauser-Horne-Shimony-Holt) inequalities [44,45]. In Ref. [46], it has been demonstrated that the BB84 family cannot be reproduced by shared classical randomness of dimension $d_{\lambda} \leq 3$. On the other hand, the BB84 family can be reproduced by performing appropriate quantum measurements on $2 \otimes 2$ quantum states [13]. Hence, each of the $P_{\lambda}^{S v}(b c \mid y z)$ is superlocal in the range $0<\mu \leq \frac{1}{\sqrt{2}}$.

For the fully local Svetlichny family $\left(0<\mu \leq \frac{1}{\sqrt{2}}\right)$, the decomposition (A.1) defines a classical simulation protocol with different deterministic distributions $P_{\lambda}^{S v}(a \mid x)$ at Alice's side, where Alice shares hidden variable $\lambda_{A-B C}$ of dimension 4 with Bob-Charlie as in Fig. 1. Decomposition (A.1) represents 2-local form across the bipartite cut $(A \mid B C)$ of the fully local Svetlichny family $\left(0<\mu \leq \frac{1}{\sqrt{2}}\right)$.

$\ddagger$ Throughout the paper we will follow the same convention 


\section{Appendix B. Demonstrating impossibility to reproduce fully local noisy Svetlichny family in the scenario where Alice preshares the hidden variable $\lambda_{A-B C}$ of dimension 2 with Bob-Charlie and uses different deterministic strategies for each $\lambda_{A-B C}$.}

Let us try to reproduce the fully local noisy Svetlichny family $P_{S v F}^{\mu}\left(0<\mu \leq \frac{1}{\sqrt{2}}\right)$ in the scenario as in Fig. 1 where Alice preshares the hidden variable $\lambda_{A-B C}$ of dimension 2 with Bob-Charlie and she uses different deterministic strategies for each $\lambda_{A-B C}$. In this case, we assume that the fully local noisy Svetlichny family $P_{S v F}^{\mu}$ $\left(0<\mu \leq \frac{1}{\sqrt{2}}\right)$ can be decomposed in the following way:

$$
P_{S v F}^{\mu}=\sum_{\lambda=0}^{1} p_{\lambda} P_{\lambda}^{S v}(a \mid x) P_{\lambda}^{S v}(b c \mid y z) .
$$

Here, $p_{0}=x_{0}, p_{1}=x_{1}\left(0<x_{0}<1,0<x_{1}<1, x_{0}+x_{1}=1\right)$. Since Alice's strategy is deterministic one, each of the two probability distributions $P_{0}^{S v}(a \mid x)$ and $P_{1}^{S v}(a \mid x)$ must be equal to any one among $P_{D}^{00}, P_{D}^{01}, P_{D}^{10}$ and $P_{D}^{11}$. In order to satisfy the marginal probabilities for Alice $P_{S v F}(a \mid x)=\frac{1}{2} \forall a, x$, the only two possible choices of $P_{\lambda}^{S v}(a \mid x)$ are:

1) $P_{D}^{00}$ and $P_{D}^{01}$ with $x_{0}=x_{1}=\frac{1}{2}$

2) $P_{D}^{10}$ and $P_{D}^{11}$ with $x_{0}=x_{1}=\frac{1}{2}$.

In case of the first choice, let us assume that $P_{0}^{S v}(a \mid x)=P_{D}^{00}, P_{1}^{S v}(a \mid x)=P_{D}^{01}$; $P_{0}^{S v}(b c \mid y z)$ and $P_{1}^{S v}(b c \mid y z)$ are given by,

$$
P_{0}^{S v}(b c \mid y z):=\left(\begin{array}{llll}
u_{11} & u_{12} & u_{13} & u_{14} \\
u_{21} & u_{22} & u_{23} & u_{24} \\
u_{31} & u_{32} & u_{33} & u_{34} \\
u_{41} & u_{42} & u_{43} & u_{44}
\end{array}\right),
$$

where $0 \leq u_{i j} \leq 1 \forall i, j$, and $\sum_{j} u_{i j}=1 \forall i$, and

$$
P_{1}^{S v}(b c \mid y z):=\left(\begin{array}{llll}
w_{11} & w_{12} & w_{13} & w_{14} \\
w_{21} & w_{22} & w_{23} & w_{24} \\
w_{31} & w_{32} & w_{33} & w_{34} \\
w_{41} & w_{42} & w_{43} & w_{44}
\end{array}\right),
$$

where $0 \leq w_{i j} \leq 1 \forall i, j$, and $\sum_{j} w_{i j}=1 \forall i$.

Now, with this choice, the box $P_{S v F}^{\mu}$ given by the model (B.1) has 
Operational nonclassicality of local multipartite correlations

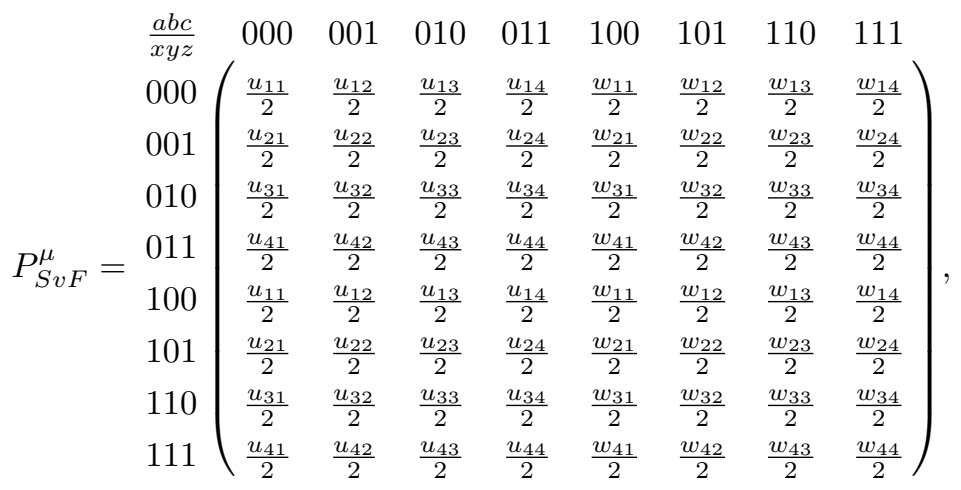

where each row and column corresponds to a fixed measurement $(x y z)$ and a fixed outcome $(a b c)$ respectively.

From Eq. (B.2), it can be seen that

$$
P_{S v F}^{\mu}(a b c \mid 001)=P_{S v F}^{\mu}(a b c \mid 101),
$$

which is not true for the fully local Svetlichny family as given in Eq. (26) with $0<\mu \leq \frac{1}{\sqrt{2}}$. Because, in case of fully local $P_{S v F}^{\mu}\left(0<\mu \leq \frac{1}{\sqrt{2}}\right)$ given in Eq. (26),

$$
P_{S v F}^{\mu}(a b c \mid 001)=\frac{2+(-1)^{a \oplus b \oplus c \sqrt{2} \mu}}{16}
$$

and

$$
P_{S v F}^{\mu}(a b c \mid 101)=\frac{2+(-1)^{a \oplus b \oplus c \oplus 1 \sqrt{2} \mu}}{16} .
$$

Again, from Eq. (B.2), it can be seen that

$$
P_{S v F}^{\mu}(a b c \mid 010)=P_{S v F}^{\mu}(a b c \mid 110),
$$

which is not true for the fully local Svetlichny family as given in Eq. (26) with $0<\mu \leq \frac{1}{\sqrt{2}}$. Because, in case of fully local $P_{S v F}^{\mu}\left(0<\mu \leq \frac{1}{\sqrt{2}}\right)$ given in Eq. (26),

$$
P_{S v F}^{\mu}(a b c \mid 010)=\frac{2+(-1)^{a \oplus b \oplus c \sqrt{2} \mu}}{16}
$$

and

$$
P_{S v F}^{\mu}(a b c \mid 110)=\frac{2+(-1)^{a \oplus b \oplus c \oplus 1} \sqrt{2} \mu}{16} .
$$

Hence, in this case, though the marginal probabilities for Alice $P_{S v F}(a \mid x)$ are satisfied, all the tripartite joint probability distributions $P_{S v F}^{\mu}$ are not satisfied simultaneously.

Similarly, in case of the first choice, if we assume that $P_{0}^{S v}(a \mid x)=P_{D}^{01}$, $P_{1}^{S v}(a \mid x)=P_{D}^{00}$, then the marginal probabilities for Alice $P_{S v F}(a \mid x)$ are satisfied, but all the tripartite joint probability distributions $P_{S v F}^{\mu}$ are not satisfied simultaneously. 
Now, in case of the second choice, let us assume that $P_{0}^{S v}(a \mid x)=P_{D}^{10}, P_{1}^{S v}(a \mid x)=$ $P_{D}^{11} ; P_{0}^{S v}(b c \mid y z)$ and $P_{1}^{S v}(b c \mid y z)$ are given by,

$$
P_{0}^{S v}(b c \mid y z)=\left(\begin{array}{cccc}
u_{11}^{\prime} & u_{12}^{\prime} & u_{13}^{\prime} & u_{14}^{\prime} \\
u_{21}^{\prime} & u_{22}^{\prime} & u_{23}^{\prime} & u_{24}^{\prime} \\
u_{31}^{\prime} & u_{32}^{\prime} & u_{33}^{\prime} & u_{34}^{\prime} \\
u_{41}^{\prime} & u_{42}^{\prime} & u_{43}^{\prime} & u_{44}^{\prime}
\end{array}\right),
$$

where $0 \leq u_{i j}^{\prime} \leq 1 \forall i, j$, and $\sum_{j} u_{i j}^{\prime}=1 \forall i$, and

$$
P_{1}^{S v}(b c \mid y z)=\left(\begin{array}{cccc}
w_{11}^{\prime} & w_{12}^{\prime} & w_{13}^{\prime} & w_{14}^{\prime} \\
w_{21}^{\prime} & w_{22}^{\prime} & w_{23}^{\prime} & w_{24}^{\prime} \\
w_{31}^{\prime} & w_{32}^{\prime} & w_{33}^{\prime} & w_{34}^{\prime} \\
w_{41}^{\prime} & w_{42}^{\prime} & w_{43}^{\prime} & w_{44}^{\prime}
\end{array}\right) \text {, }
$$

where $0 \leq w_{i j}^{\prime} \leq 1 \forall i, j$, and $\sum_{j} w_{i j}^{\prime}=1 \forall i$.

Now, with this choice, the box $P_{S v F}^{\mu}\left(0<\mu \leq \frac{1}{\sqrt{2}}\right)$ given by the model (B.1) has,

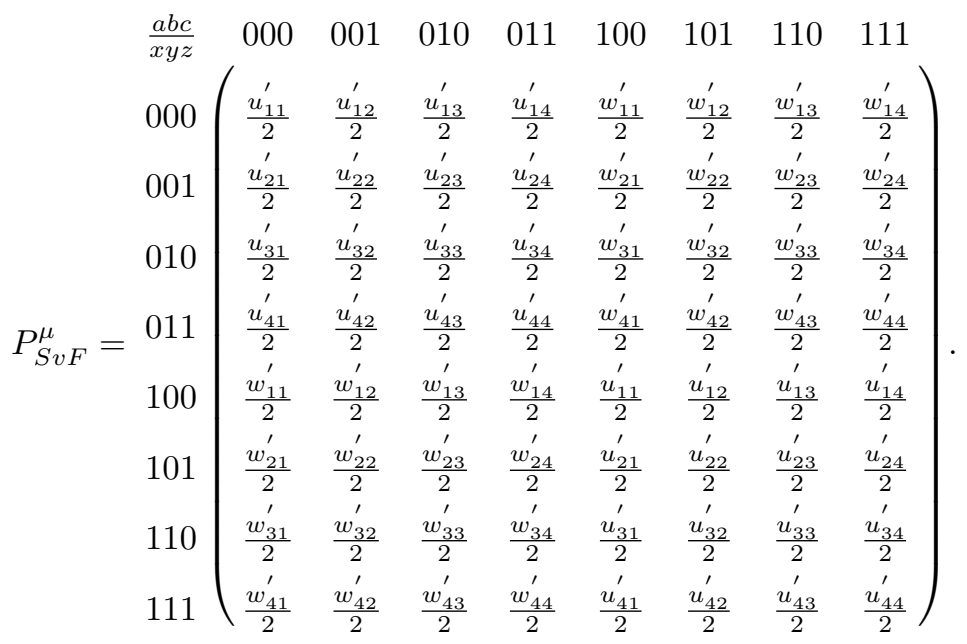

From Eq. (B.3), it can be seen that

$$
P_{S v F}^{\mu}(0 b c \mid 000)=P_{S v F}^{\mu}(1 b c \mid 100),
$$

which is not true for the fully local Svetlichny family as given in Eq. (26) with $0<\mu \leq \frac{1}{\sqrt{2}}$. Because, in case of fully local $P_{S v F}^{\mu}\left(0<\mu \leq \frac{1}{\sqrt{2}}\right)$ given in Eq. (26),

$$
P_{S v F}^{\mu}(0 b c \mid 000)=\frac{2+(-1)^{b \oplus c} \sqrt{2} \mu}{16}
$$

and

$$
P_{S v F}^{\mu}(1 b c \mid 100)=\frac{2+(-1)^{b \oplus c \oplus 1} \sqrt{2} \mu}{16} .
$$

From Eq. (B.3), it can be seen that

$$
P_{S v F}^{\mu}(1 b c \mid 000)=P_{S v F}^{\mu}(0 b c \mid 100),
$$


which is not true for the fully local Svetlichny family as given in Eq. (26) for $0<\mu \leq \frac{1}{\sqrt{2}}$. Because, in case of fully local $P_{S v F}^{\mu}\left(0<\mu \leq \frac{1}{\sqrt{2}}\right)$ as given in Eq. $(26)$,

$$
P_{S v F}^{\mu}(1 b c \mid 000)=\frac{2+(-1)^{b \oplus c \oplus 1 \sqrt{2} \mu}}{16}
$$

and

$$
P_{S v F}^{\mu}(0 b c \mid 100)=\frac{2+(-1)^{b \oplus c} \sqrt{2} \mu}{16} .
$$

Again, from Eq. (B.3), it can be seen that

$$
P_{S v F}^{\mu}(0 b c \mid 011)=P_{S v F}^{\mu}(1 b c \mid 111),
$$

which is not true for fully local $P_{S v F}^{\mu}$ as given in Eq. (26) for $0<\mu \leq \frac{1}{\sqrt{2}}$. Because, in case of fully local $P_{S v F}^{\mu}\left(0<\mu \leq \frac{1}{\sqrt{2}}\right)$ as given in Eq. (26),

$$
P_{S v F}^{\mu}(0 b c \mid 011)=\frac{2+(-1)^{b \oplus c \oplus 1} \sqrt{2} \mu}{16}
$$

and,

$$
P_{S v F}^{\mu}(1 b c \mid 111)=\frac{2+(-1)^{b \oplus c} \sqrt{2} \mu}{16}
$$

From Eq. (B.3), it can be seen that

$$
P_{S v F}^{\mu}(1 b c \mid 011)=P_{S v F}^{\mu}(0 b c \mid 111),
$$

which is not true for fully local $P_{S v F}^{\mu}$ as given in Eq. (26) for $0<\mu \leq \frac{1}{\sqrt{2}}$. Because, in case of fully local $P_{S v F}^{\mu}\left(0<\mu \leq \frac{1}{\sqrt{2}}\right)$ as given in Eq. (26),

$$
P_{S v F}^{\mu}(1 b c \mid 011)=\frac{2+(-1)^{b \oplus c} \sqrt{2} \mu}{16}
$$

and

$$
P_{S v F}^{\mu}(0 b c \mid 111)=\frac{2+(-1)^{b \oplus c \oplus 1} \sqrt{2} \mu}{16} .
$$

Hence, in this case, though the marginal probabilities for Alice $P_{S v F}(a \mid x)$ are satisfied, all the tripartite joint probability distributions $P_{S v F}^{\mu}$ are not satisfied simultaneously.

Similarly, in case of the second choice, if we assume that $P_{0}^{S v}(a \mid x)=P_{D}^{11}$, $P_{1}^{S v}(a \mid x)=P_{D}^{10}$, then the marginal probabilities for Alice $P_{S v F}(a \mid x)$ are satisfied, but all the tripartite joint probability distributions $P_{S v F}^{\mu}$ are not satisfied simultaneously.

Note that this proof is valid for any two bipartite correlations $P_{\lambda}^{S v}(b c \mid y z)$ $(\lambda=0,1)$ shared between Bob and Charlie at each $\lambda_{A-B C}$ without any constraint on the correlations. Hence, it is obvious that this proof will also be valid when these two bipartite correlations $P_{\lambda}^{S v}(b c \mid y z)(\lambda=0,1)$ at Bob-Charlie's side are NS boxes and satisfy the marginal probabilities $P_{S v F}(b \mid y), P_{S v F}(c \mid z)$ at Bob and Charlie's side, 
respectively.

It is, therefore, impossible to reproduce the fully local box $P_{S v F}^{\mu}\left(0<\mu \leq \frac{1}{\sqrt{2}}\right)$ in the scenario as in Fig. 1 where Alice preshares the hidden variable $\lambda_{A-B C}$ of dimension 2 with Bob-Charlie.

Appendix C. 2-local form across the bipartition $(A \mid B C)$ for the fully local Mermin family $P_{M F}^{\nu}$ in the range $0<\nu \leq \frac{1}{2}$

For $0<\nu \leq \frac{1}{2}$ the fully local noisy Mermin box can be decomposed in a convex mixture of the 3-local deterministic boxes. In this range, we consider the following decomposition for the Mermin family in terms of the 3-local deterministic boxes:

$$
\begin{aligned}
& P_{M F}^{\nu}=\frac{1}{4} P_{D}^{00}\left\{\frac { 2 \nu } { 8 } \left(P_{D}^{0000}+P_{D}^{0010}+P_{D}^{0101}+P_{D}^{0111}\right.\right. \\
& \left.+P_{D}^{1000}+P_{D}^{1011}+P_{D}^{1101}+P_{D}^{1110}\right) \\
& \left.+\frac{1-2 \nu}{4}\left(P_{D}^{1000}+P_{D}^{1100}+P_{D}^{1101}+P_{D}^{1001}\right)\right\} \\
& +\frac{1}{4} P_{D}^{01}\left\{\frac { 2 \nu } { 8 } \left(P_{D}^{0001}+P_{D}^{0011}+P_{D}^{0100}+P_{D}^{0110}\right.\right. \\
& \left.+P_{D}^{1001}+P_{D}^{1010}+P_{D}^{1100}+P_{D}^{1111}\right) \\
& \left.+\frac{1-2 \nu}{4}\left(P_{D}^{1000}+P_{D}^{1100}+P_{D}^{1101}+P_{D}^{1001}\right)\right\} \\
& +\frac{1}{4} P_{D}^{01}\left\{\frac { 2 \nu } { 8 } \left(P_{D}^{0000}+P_{D}^{0011}+P_{D}^{0101}+P_{D}^{0110}\right.\right. \\
& \left.+P_{D}^{1100}+P_{D}^{1001}+P_{D}^{1110}+P_{D}^{1011}\right) \\
& \left.+\frac{1-2 \nu}{4}\left(P_{D}^{1000}+P_{D}^{1100}+P_{D}^{1101}+P_{D}^{1001}\right)\right\} \\
& +\frac{1}{4} P_{D}^{01}\left\{\frac { 2 \nu } { 8 } \left(P_{D}^{0001}+P_{D}^{0010}+P_{D}^{0100}+P_{D}^{0111}\right.\right. \\
& \left.+P_{D}^{1000}+P_{D}^{1010}+P_{D}^{1101}+P_{D}^{1111}\right) \\
& \left.+\frac{1-2 \nu}{4}\left(P_{D}^{1000}+P_{D}^{1100}+P_{D}^{1101}+P_{D}^{1001}\right)\right\} \\
& :=\sum_{\lambda=0}^{3} r_{\lambda} P_{\lambda}^{M}(a \mid x) P_{\lambda}^{M}(b c \mid y z),
\end{aligned}
$$


where $r_{0}=r_{1}=r_{2}=r_{3}=\frac{1}{4}$;

$$
\begin{aligned}
& P_{0}^{M}(a \mid x)=P_{D}^{00}, P_{1}^{M}(a \mid x)=P_{D}^{01}, \\
& P_{2}^{M}(a \mid x)=P_{D}^{10}, P_{3}^{M}(a \mid x)=P_{D}^{11}
\end{aligned}
$$

and

$$
\begin{aligned}
& P_{0}^{M}(b c \mid y z)=\left\{\frac { 2 \nu } { 8 } \left(P_{D}^{0000}+P_{D}^{0010}+P_{D}^{0101}+P_{D}^{0111}\right.\right. \\
& \left.+P_{D}^{1000}+P_{D}^{1011}+P_{D}^{1101}+P_{D}^{1110}\right) \\
& \left.+\frac{1-2 \nu}{4}\left(P_{D}^{1000}+P_{D}^{1100}+P_{D}^{1101}+P_{D}^{1001}\right)\right\} \\
& =\left(\begin{array}{cccc}
\frac{1+\nu}{4} & \frac{1-\nu}{4} & \frac{1-\nu}{4} & \frac{1+\nu}{4} \\
\frac{1+\nu}{4} & \frac{1-\nu}{4} & \frac{1-\nu}{4} & \frac{1+\nu}{4} \\
\frac{1+\nu}{4} & \frac{1-\nu}{4} & \frac{1-\nu}{4} & \frac{1+\nu}{4} \\
\frac{1-\nu}{4} & \frac{1+\nu}{4} & \frac{1+\nu}{4} & \frac{1-\nu}{4}
\end{array}\right), \\
& P_{1}^{M}(b c \mid y z)=\left(\begin{array}{cccc}
\frac{1-\nu}{4} & \frac{1+\nu}{4} & \frac{1+\nu}{4} & \frac{1-\nu}{4} \\
\frac{1-\nu}{4} & \frac{1+\nu}{4} & \frac{1+\nu}{4} & \frac{1-\nu}{4} \\
\frac{1-\nu}{4} & \frac{1+\nu}{4} & \frac{1+\nu}{4} & \frac{1-\nu}{4} \\
\frac{1+\nu}{4} & \frac{1-\nu}{4} & \frac{1-\nu}{4} & \frac{1+\nu}{4}
\end{array}\right), \\
& P_{2}^{M}(b c \mid y z)=\left(\begin{array}{cccc}
\frac{1-\nu}{4} & \frac{1+\nu}{4} & \frac{1+\nu}{4} & \frac{1-\nu}{4} \\
\frac{1+\nu}{4} & \frac{1-\nu}{4} & \frac{1-\nu}{4} & \frac{1+\nu}{4} \\
\frac{1+\nu}{4} & \frac{1-\nu}{4} & \frac{1-\nu}{4} & \frac{1+\nu}{4} \\
\frac{1+\nu}{4} & \frac{1-\nu}{4} & \frac{1-\nu}{4} & \frac{1+\nu}{4}
\end{array}\right), \\
& P_{3}^{M}(b c \mid y z)=\left(\begin{array}{cccc}
\frac{1+\nu}{4} & \frac{1-\nu}{4} & \frac{1-\nu}{4} & \frac{1+\nu}{4} \\
\frac{1-\nu}{4} & \frac{1+\nu}{4} & \frac{1+\nu}{4} & \frac{1-\nu}{4} \\
\frac{1-\nu}{4} & \frac{1+\nu}{4} & \frac{1+\nu}{4} & \frac{1-\nu}{4} \\
\frac{1-\nu}{4} & \frac{1+\nu}{4} & \frac{1+\nu}{4} & \frac{1-\nu}{4}
\end{array}\right) .
\end{aligned}
$$

Note that, each of the probability distributions $P_{\lambda}^{M}(b c \mid y z)$ is local for $0<\nu \leq \frac{1}{2}$, as they satisfy the complete set of Bell-CHSH inequalities $[44,45]$ in this range. In fact, each of the $P_{\lambda}^{M}(b c \mid y z)$ is superlocal in the range $0<\nu \leq \frac{1}{2}$. Because each of the $P_{\lambda}^{M}(b c \mid y z)$ belongs to CHSH family [13],

$$
P_{C H S H}(b c \mid y z)=\frac{2+(-1)^{b \oplus c \oplus y z} \sqrt{2} V}{8}
$$

with $0<V=\sqrt{2} \nu \leq 1$, upto local reversible operations. In Ref. [14], it has been shown that the above local box is superlocal for any $V>0$.

For the fully local noisy Mermin-box $\left(0<\nu \leq \frac{1}{2}\right)$, the decomposition (C.1) defines a classical simulation protocol with different deterministic distributions $P_{\lambda}^{M}(b c \mid y z)$ at Alice's side, where Alice shares hidden variable $\lambda_{A-B C}$ of dimension 4 as in Fig. 1. Decomposition (C.1) represents 2-local form across the bipartite cut $(A \mid B C)$ of the fully local noisy Mermin-box $\left(0<\nu \leq \frac{1}{2}\right)$. 


\section{Appendix D. 2-local form across the bipartition $(A \mid B C)$ for the Mermin family $P_{M F}^{\nu}$ in the range $0<\nu \leq 1$}

For $0<\nu \leq 1$ the 2-local Mermin family can be decomposed in a convex mixture of the local vertices and 2-local vertices as follows:

$$
\begin{aligned}
P_{M F}^{0<\nu<1} & =\frac{1}{4} P_{D}^{00}(a \mid x)\left(\nu P_{P R}^{000}(b c \mid y z)+\frac{1-\nu}{16} \sum_{\gamma, \epsilon, \zeta, \eta} P_{D}^{\gamma \epsilon \zeta \eta}(b c \mid y z)\right) \\
& +\frac{1}{4} P_{D}^{01}(a \mid x)\left(\nu P_{P R}^{001}(b c \mid y z)+\frac{1-\nu}{16} \sum_{\gamma, \epsilon, \zeta, \eta} P_{D}^{\gamma \epsilon \zeta \eta}(b c \mid y z)\right) \\
& +\frac{1}{4} P_{D}^{10}(a \mid x)\left(\nu P_{P R}^{111}(b c \mid y z)+\frac{1-\nu}{16} \sum_{\gamma, \epsilon, \zeta, \eta} P_{D}^{\gamma \epsilon \zeta \eta}(b c \mid y z)\right) \\
& +\frac{1}{4} P_{D}^{11}(a \mid x)\left(\nu P_{P R}^{110}(b c \mid y z)+\frac{1-\nu}{16} \sum_{\gamma, \epsilon, \zeta, \eta} P_{D}^{\gamma \epsilon \zeta \eta}(b c \mid y z)\right) \\
& :=\sum_{\lambda=0}^{3} r_{\lambda} P_{\lambda}^{M}(a \mid x) P_{\lambda}^{M}(b c \mid y z),
\end{aligned}
$$

where $r_{0}=r_{1}=r_{2}=r_{3}=\frac{1}{4}$;

$$
\begin{aligned}
& P_{0}^{M}(a \mid x)=P_{D}^{00}, P_{1}^{M}(a \mid x)=P_{D}^{01}, \\
& P_{2}^{M}(a \mid x)=P_{D}^{10}, P_{3}^{M}(a \mid x)=P_{D}^{11}
\end{aligned}
$$

and

$$
\begin{aligned}
& P_{0}^{M}(b c \mid y z)=\nu P_{P R}^{000}(b c \mid y z)+\frac{1-\nu}{16} \sum_{\gamma, \epsilon, \zeta, \eta} P_{D}^{\gamma \epsilon \zeta \eta}(b c \mid y z), \\
& P_{1}^{M}(b c \mid y z)=\nu P_{P R}^{001}(b c \mid y z)+\frac{1-\nu}{16} \sum_{\gamma, \epsilon, \zeta, \eta} P_{D}^{\gamma \epsilon \zeta \eta}(b c \mid y z), \\
& P_{2}^{M}(b c \mid y z)=\nu P_{P R}^{111}(b c \mid y z)+\frac{1-\nu}{16} \sum_{\gamma, \epsilon, \zeta, \eta} P_{D}^{\gamma \epsilon \zeta \eta}(b c \mid y z), \\
& P_{3}^{M}(b c \mid y z)=\nu P_{P R}^{110}(b c \mid y z)+\frac{1-\nu}{16} \sum_{\gamma, \epsilon, \zeta, \eta} P_{D}^{\gamma \epsilon \zeta \eta}(b c \mid y z) .
\end{aligned}
$$

Note that, each of the probability distributions $P_{\lambda}^{M}(b c \mid y z)$ satisfy NS principle for $0<\nu \leq 1$, Hence, the noisy Mermin box has the 2-local form across the bipartite cut $(A \mid B C)$ in the range $0<\nu \leq 1$. For the 2-local noisy Mermin-box $(0<\nu \leq 1)$, the decomposition (D.1) defines a classical simulation protocol as in Fig. 2 where Alice shares hidden variable $\lambda_{A-B C}$ of dimension 4 . 